\title{
Viertes Kapitel Wittgenstein über logische Operationen
}

Sofern die mit dem Bild ausführbaren Operationen als ihre Basen und Resultate Symbole haben, können sie als symbolische Operationen bezeichnet werden. Die in dem letzten Abschnitt definierte Problematik der Bildtheorie legt nahe, dass symbolische Operationen sich nicht auf logische Operationen, welche sogenannte Wahrheitsoperationen und Operationen des logischen Schließens umfassen, beschränken, sondern weitere die Komponenten der logischen Operationen bildende oder Voraussetzungen für ihre Anwendung schaffende Operationen einschließen, die ihrerseits auf Symbole, die keine Sätze sind, angewandt werden. Obwohl erst die Bestimmung des Charakters der Operationen der letzteren Art den Übergang Wittgensteins von der Theorie des Tractatus zu seiner Spätphilosophie erlaubt, besteht die vorrangige Rolle des Begriffs einer logischen Operation in diesem Übergang darin, dass ohne seine Charakterisierung die Unterscheidung logischer Operationen als einer besonderen Art symbolischer Operationen nicht möglich ist.

$\S 1$. Wittgensteins Begriff der Operation und der Begriff der Operation in der algebraisch-logischen Tradition

1965 bewertet Richter Wittgensteins Auffassung der Logik im Tractatus als eine operative Auffassung, die sich dadurch auszeichnet, dass Logik als $\tau \varepsilon ́ \chi v \eta$ und nicht als Lehre, die von besonderen logischen Gegenständen handelt, verstanden wird ${ }^{1}$. Diese Auffassung drückt sich in erster Linie in dem Begriff der Operation aus, der bei Wittgenstein nach Meinung Sundholms (1992) eine zweifache Auslegung zulässt: Man kann unter einer Operation eine Vorschrift, die eine Handlung fordert, einerseits und eine

\footnotetext{
${ }^{1}$ V. Richter. Untersuchungen zur operativen Logik der Gegenwart. Freiburg, München, Verlag Karl Alber, 1965, 11, 27, 30, 37-38
} 
Handlung nach einer solchen Vorschrift andererseits verstehen ${ }^{2}$. Im weiteren betrachte ich Operationen als Handlungen, davon ausgehend, dass als Vorschrift ein einen Operator (eine logische Konstante) enthaltendes zusammengesetztes Symbol, z.B. ein zusammengesetzter Satz, fungiert.

Der Gebrauch des Begriffs der Operation ist in erster Linie für die Tradition der Algebra der Logik charakteristisch. Dieser Gebrauch geht auf die Entwicklung des sogenannten Kalküls der Operationen und der symbolischen Algebra in Großbritannien in der ersten Hälfte des 19ten Jahrhunderts durch Mathematiker der Universität Cambridge wie Woodhouse, Peacock, Babbage und Herschel zurück ${ }^{3}$. Die als eine Antwort auf die Entwicklung der mathematischen Analysis auf dem Kontinent geltende Arbeit der Mathematiker dieses Kreises mündet in eine neue Auffassung der Algebra in den Werken von Peacock, Gregory, De Morgan und Boole ${ }^{4}$. Charakteristische Merkmale der Behandlung von Operationen in der algebraisch-logischen Tradition sind folgende:

1. Operationen werden als Handlungen oder Verfahren betrachtet ${ }^{5}$. Als Objekte solcher Handlungen treten Symbole und insbesondere Zahlensymbole auf ${ }^{6}$. Handlungen mit Symbolen werden als real ausführbare Handlungen aufgefasst ${ }^{7}$, die als Produkt ihrer Anwendung ein neues Objekt, nämlich ein neues Symbol, ergeben ${ }^{8}$.

2. Obwohl zum Untersuchungsgegenstand der Algebra der Logik Handlungen mit Symbolen gehören, sind solche Handlungen stellvertretend für Operationen, die als das von den Operationssymbolen

\footnotetext{
${ }^{2}$ G. Sundholm. „The General Form of the Operation in Wittgenstein's Tractatus“ (im weiteren: Sundholm.Form). In: Grazer philosophische Studien 42, 1992, 57-76, 59

${ }^{3}$ I. Grattan-Guiness (Hrsg.). Companion Encyclopedia of the History and Philosophy of Mathematical Sciences. London, New York, Routledge, 1994, 800

${ }^{4}$ E. Koppelman. "The Calculus of Operations and the Rise of Abstract Algebra" (im weiteren: Koppelman). In: Archive for History of Exact Sciences 8, 1971-72, 155-242, 156

${ }^{5}$ Ebd., 195

${ }^{6}$ E. Schröder. Der Operationskreis des Logikkalkuls (im weiteren: Schröder.Operationskreis). Darmstadt, Wissenschaftliche Buchgesellschaft, 1966, 2

${ }^{7}$ A. Cayley. "On the Notion and Boundaries of Algebra". In: The Collected Papers of A. Cayley V. Cambridge, University Press, 1892, 292-294, 293

${ }^{8}$ Schröder.Operationskreis, 2, 13
} 
Dargestellte $^{9}$ oder als ihr Substrat charakterisiert wird ${ }^{10}$. Dieses Substrat wird einerseits in den Operationen des Geistes auf Objekten des Denkens und Argumentierens ${ }^{11}$, insbesondere auf Klassen ${ }^{12}$, und andererseits in solchen anschaulichen Handlungen wie den geometrischen $^{13}$ gesehen. Die Funktion des Substrats der symbolischen Handlungen sieht Schröder in der Bestimmung des Zwecks, welchen Operationen außer ihrem Resultat haben. Ein solcher Zweck wird definiert, indem definiert wird, was durch die Ausführung einer Operation auf Objekten, die durch Symbole der Basen der Operation repräsentiert werden, erreicht wird. So ist der Zweck der Multiplikation eine „Determination“, die darin besteht, dass ein gewisser Begriff eine nähere Bestimmung durch die Forderung erfährt, dass die mit diesem Begriff gemeinten Gegenstände auch die Merkmale der Gegenstände eines anderen Begriffs haben sollen ${ }^{14}$. Was Schröder als Zweck der Operation betrachtet, ist nach Boole das von dem Resultat der Operation Dargestellte ${ }^{15}$.

3. Operationen haben Beziehungen zu einander ${ }^{16}$. Diese Beziehungen realisieren sich dadurch, dass Operationen mit einander nach gewissen Gesetzen kombiniert werden und inverse Operationen haben können, sowie durch andere Operationen definierbar und ersetzbar sind. Operationen können komplex (zusammengesetzt) in dem Sinn sein, dass sie mehrere Operationen einschließen ${ }^{17}$.

4. Jede Operation und jede Kombination von Operationen unterliegt bestimmten allgemeinen Gesetzen ${ }^{18}$, durch deren Angabe Operationen mit Symbolen definiert werden. Erst diese Angabe gewährleistet den

\footnotetext{
${ }^{9}$ D.F. Gregory. „On a Difficulty in the Theory of Algebra” (im weiteren: Gregory). In: The Cambridge Mathematical Journal 3, 1842, 153-159, 153-154

${ }^{10}$ Schröder.Operationskreis, 5

${ }^{11}$ Ch.S. Peirce. "On the Algebra of Logic: A Contribution to the Philosophy of Notation". In: American Journal of Mathematics 7, 1885, 180-202, 182

${ }^{12}$ Boole.Analysis, 51

${ }^{13}$ Schröder.Operationskreis, 5

${ }^{14}$ Ebd., 6, 33

${ }^{15}$ Boole.Analysis, 61

${ }^{16}$ Ebd., 51

${ }^{17}$ Schröder.Operationskreis, 2-4, Boole.Analysis, 61

${ }^{18}$ Boole. Analysis, 51
} 
darstellenden Charakter des Symbols der Operation ${ }^{19}$. Dass als Basen von Operationen Symbole und ihre Kombinationen definiert werden, bedeutet, dass die die Operationen definierenden Gesetze nicht von einer bestimmten Interpretation von Symbolen abhängen, sondern verschiedene Interpretationen erlauben. Deswegen sind diese Gesetze allgemein ${ }^{20}$. Die Allgemeinheit dieser Gesetze ist der Grund für die Formulierung der Idee der Konstruktion allgemeiner uninterpretierter Kalküle von Operationen, in welchen die Gesetze des Operierens mit Symbolen die Form interpretierbarer Funktionsgleichungen haben, so dass der Kalkül, der eine logisch zusammenhängende Gesamtheit solcher Gesetze ist, durch eine Interpretation zum Kalkül von bestimmten Operationen werden kann ${ }^{21}$.

5. Funktionsausdrücke werden gebraucht, um die Operationsschreibweise $\mathrm{zu}$ verallgemeinern und so die Möglichkeit zu erlangen, gesetzmäßige Beziehungen zwischen Operationen zu charakterisieren, ohne diese oder jene Operation durch ihr Symbol anzugeben ${ }^{22}$. Die Besonderheit dieses Gebrauchs besteht darin, dass der Funktionsausdruck als allgemeine Bezeichnung des Resultats der Anwendung einer Operation dient ${ }^{23}$ : An der Argumentstelle des Funktionszeichens wird das Symbol angegeben, auf welches die Operation angewandt wird. Schröder benutzt diese Besonderheit der allgemeinen Darstellung von Operationen, um die für Operationen geltenden Gesetze symbolisch darzustellen $^{24}$. Gelten für verschiedene Operationen dieselben Gesetze, werden die Gesetze mittels der Funktionszeichen auf eine einheitliche Weise dargestellt. Der Begriff der Funktion wird zugleich für die Bezeichnung eines Ausdrucks benutzt, dessen Be-

${ }^{19}$ Gregory, 155

${ }^{20}$ Boole.Analysis, 49-50

${ }^{21}$ E. Schröder. „Ueber Algorithmen und Calculn“ (im weiteren: Schröder.Algorithmen). In: Archiv der Mathematik und Physik 5, 1887, 225-278, 226, 228 229

22 Gregory, 154

${ }^{23}$ Koppelman weist darauf hin, dass Operationenkalkül von den Begründern der symbolischen Algebra und insbesondere von De Morgan als ein Teil des Funktionenkalküls behandelt wurde, weil man in Operationen ein Mittel der Zurückführung von bestimmten gegebenen Funktionen auf die mit ihnen identischen Funktionen sah. Koppelman, 209

${ }^{24}$ Schröder.Algorithmen, 228 
standteile Symbole an der Argumentstelle des Funktionszeichens sowie ihre Kombinationen sind ${ }^{25}$.

6. Auch durch die Operationssymbole verbundene und die Objekte, mit welchen operiert wird, andeutende Variablen wie $\mathrm{x}$ werden als Operationssymbole aufgefasst. Nach Meinung Gregorys wurde diese Auffassung durch den Übergang in der Schreibweise für die Operation der Multiplikation von der Angabe des Multiplikationszeichens zum Weglassen dieses Zeichens verursacht. Dank diesem Weglassen fing man an, die Faktoren des Produkts selbst als Operationssymbole zu behandeln, was letztendlich die Bezeichnung ,symbolische Algebra" für die Theorie, welche allgemeine Gesetze der Kombination von Operationen formuliert, rechtfertigte ${ }^{26}$. Für die Zahlen bedeutet diese Betrachtungsweise, dass jede natürliche Zahl $n$ als eine $n$-fache Anwendung einer Operation (der Operation des Nehmens von 1) verstanden wird ${ }^{27}$. Boole, der zwischen Symbolen, welche einzelne Individuen oder Sachverhalte darstellen, und Auswahlsymbolen, d.h. Symbolen der Operation der Klassenbildung, die auf Gruppen von Individuen operieren, unterscheidet ${ }^{28}$, bezeichnet das Gesetz, das zusammen mit anderen Gesetzen die Operation der Klassenbildung charakterisiert und das die Auswahlsymbole als Elemente eines logischen Kalküls von den Elementen eines mathematischen Kalküls unterscheidet, als Index-Gesetz, das besagt, dass iterative Anwendung derselben Operation als ihr Resultat dasselbe Symbol ergibt, wie ihre einmalige Anwendung. Bezeichnet wird die iterative Anwendung einer Operation analog dem Potenzieren ${ }^{29}$.

\footnotetext{
${ }^{25}$ Boole.Analysis, 61

${ }^{26}$ Gregory, 158

${ }^{27}$ S. Bryant. "The Relation of Mathematics to General Formal Logic”. In: Proceedings of the Aristotelian Society 2, 1902, 105-134, 109

${ }^{28}$ Boole.Analysis, 60

${ }^{29}$ Ebd., 62-63
} 
$\S 1$ a. Konstruktive Operationen Wittgensteins

Wittgenstein spricht über logische Operationen, ohne sie in Arten zu unterteilen. Als Operationen charakterisiert er Operationen auf Sätzen. Ausdrücklich bezeichnet er als Operationen vor allem Wahrheitsoperationen, die mit Hilfe logischer Konstanten, z.B. Konjunktions- oder Disjunktionszeichen, symbolisiert werden, und die ich als konstruktive Operationen bezeichne, sowie bejahende und verneinende Operationen. Die bejahenden und verneinenden Operationen kann man als eine besondere Art von Operationen betrachten, die das logische Schließen realisieren ${ }^{30}$.

Wittgensteins Charakterisierung konstruktiver Operationen erlaubt es, ihnen folgende Merkmale zuzusprechen:

1. Konstruktive Operationen sind Handlungen, welche aus Symbolen neue Symbole hervorbringen. Als Basen und Resultate von Operationen treten Sätze auf. Als ihre Basen können Operationen Produkte ihrer eigenen Anwendung haben. Die im Tractatus eingeführte Operation der Negation (im weiteren N-Operation) ist so definiert, dass die Mengen der Sätze, auf welche sie angewandt wird, in Hinblick auf ihre Mächtigkeit nicht beschränkt sind.

2. Der Zweck konstruktiver Operationen ist es, die bestehenden oder möglichen Beziehungen zwischen Tatsachen darzustellen. Die Bedeutung logischer Konstanten, die nicht vertreten, besteht darin, dass die mit ihrer Hilfe konstruierten zusammengesetzten Sätze die logische Struktur der Welt zeigen ${ }^{31}$. Indem Wittgenstein logische Konstanten für nicht vertretende Elemente des Symbolismus und somit die mit ihrer Hilfe definierbaren Operationen für nur auf Zeichen ausführbare Operationen erklärt, bestreitet er, dass konstruktive Operationen ein Substrat haben. Ein solches Substrat kann man jedoch in einer bestimmten Beschreibung der Welt oder in der Konstruktion eines Bildes der Wirklichkeit sehen, wenn man von der Schröder-

\footnotetext{
${ }^{30}$ Die Möglichkeit, verschiedene Arten von logischen Operationen zu unterscheiden, und unter diesen als eine besondere Art die Operationen des Schließens zu behandeln, benutzt z.B. Anscombe in ihrer Argumentation. G.E.M. Anscombe. An Introduction to Wittgenstein's Tractatus (im weiteren: Anscombe.Introduction). London, Hutchinson University Library, 1971, 4. Aufl., 117

${ }^{31}$ Tagebücher, 18.10.14, TLP, 4.023
} 
schen Identifizierung des Substrats mit dem den Zweck einer Operation Bestimmenden ausgeht. Eine Beschreibung der Welt beruht auf der Annahme der Identität zwischen den logisch-syntaktischen $\mathrm{Zu}-$ sammenhängen und dem logischen Gerüst der Welt (internen Beziehungen zwischen Tatsachen). Selbst wenn die Beschreibung der Welt dieses Identische zeigt und nicht behauptet, ist das Zeigen dieses Identischen das durch die Einführung des logischen Symbolismus Bezweckte. Die Beschreibung der Welt, welche die Funktion eines solchen Zeigens erfüllt, kann als Substrat logischer Operationen angesehen werden, sofern erst das Vorhandensein der Beschreibung die Formulierung dieses Zwecks als des Zwecks der Entwicklung des logischen Symbolismus ermöglicht und somit den Zweck des Symbolismus bestimmt. Das Substrat in diesem Sinn besteht nicht aus den Operationen einer anderen Natur im Vergleich zu logischen Operationen. Es ist eher eine Menge von Sätzen, auf welche logische konstruktive Operationen angewandt werden und die sich durch jede solche Anwendung verändert, so dass die Menge, die das Produkt einer bestimmten Anwendung von Operationen ist, einen besonderen Schritt in der Konstruktion der Beschreibung der Welt darstellt.

3. Eine konstruktive Operation oder Kombination konstruktiver Operationen kann sich selbst oder eine andere konstruktive Operation aufheben, wie die Negation, die sich selbst aufhebt. In diesem Sinn kann man von Inversen konstruktiver Operationen reden. Operationen können einander ersetzen, sofern derselbe Satz durch die Anwendung verschiedener konstruktiver Operationen und der auf den Gesetzen ihrer Kombinationen beruhenden gegenseitigen Ersetzung von Zeichen gewonnen werden kann. So lässt sich der Satz „,p. q “ einerseits aus den Basen $\mathrm{p}$ und $\mathrm{q}$ durch die Verneinung von $\mathrm{q}$ und die Konjunktion von $\mathrm{p}$ mit dem Resultat der Verneinung gewinnen. Andererseits gewinnt man den Satz dadurch, dass man $\mathrm{p}$ verneint, die Disjunktion des Resultats der Verneinung mit q bildet, das Resultat der Disjunktion verneint und das gewonnene Zeichen durch ein Zeichen mit denselben Wahrheitsbedingungen ersetzt.

4. Sofern logische Operationen Wahrheitsoperationen sind und das Satzzeichen die Abzeichen „W“ und „F“ für die Wahrheitsmöglichkeiten (für die Pole) des Satzes beinhaltet, sind Gesetze, welchen 
konstruktive Operationen unterliegen, Gesetze der Zuordnung der Wahrheit und Falschheit zu den Wahrheitsmöglichkeiten der Wahrheitsargumente eines Satzes ${ }^{32}$. Angegeben werden die Zuordnungsgesetze für konstruktive Operationen in dem Symbolismus, der im Tractatus entworfen wird, durch die Konstruktionsregel (durch die Definition der allgemeinen Form des Satzes) sowie durch einige Substitutionsregeln, wie $N(p)=\sim p^{33}$.

5. Die Resultate der Anwendung konstruktiver Operationen bezeichnet Wittgenstein als Wahrheitsfunktionen. Der Funktionsausdruck, der laut den Forderungen des Tractatus ein Satzzeichen mit den Abzeichen „,W“ und „F“ sein soll, ermöglicht es, die Basen der den Ausdruck hervorbringenden Wahrheitsoperation, d.h. die Wahrheitsargumente des Satzes, zu bestimmen und vor allem festzustellen, ob das Resultat der Operation ein sinnvoller Satz, eine Tautologie oder eine Kontradiktion ist.

6. Als Exponenten einer iterativ angewandten konstruktiven Operation werden von Wittgenstein natürliche Zahlen definiert ${ }^{34}$. Jedes Zahlzeichen kann man als Resultat einer iterativen Anwendung der die Zahl definierenden Operation und zugleich als einen Funktionsausdruck betrachten, der die Anzahl der Iterationen angibt.

Diese Merkmale weisen Analogien zu den meisten die Herangehensweise der Algebra der Logik auszeichnenden Charakteristika der symbolischen Operationen auf. Für einige Thesen Wittgensteins, insbesondere für die Einführung der N-Operation sowie für die Charakterisierung des logischen Schließens als eines Übergangs von Sätzen, die mehr sagen, zu Sätzen, die weniger sagen $^{35}$, lassen sich Parallelen unter den Thesen der Vertreter der Algebra der Logik finden: So behauptet Boole, dass die Methode der Deduktion in der fortschreitenden Einschränkung besteht und dass alle zusammengesetzten Sätze als Verneinungen der Koexistenz der Wahrheit

\footnotetext{
${ }^{32}$ Bereits in den Tagebüchern definiert Wittgenstein die Verneinung durch Gesetze, welche für sie gelten: Sie wird als eine logische Operation eingeführt, die sich selbst durch die zweifache Anwendung aufhebt und deren Anwendung auf einen Satz nicht mit dem Satz selbst gleichgesetzt werden kann. Tagebücher, 13.12.14

${ }^{33}$ TLP, 5.5-5.502, 5.51, 5.52

${ }^{34}$ Ebd., 6.021

${ }^{35}$ Ebd., 5.14
} 
oder Falschheit bestimmter Behauptungen darstellbar sind, was die Verneinung zur wesentlichen Konstituente eines jeden zusammengesetzten Satzes macht ${ }^{36}$.

\section{$\S 1$ b. Zu Geach-Fogelin Diskussion}

Sofern konstruktive Operationen den Bestand der logischen Notation und insbesondere die Menge aller mit den notationalen Mitteln konstruierbaren Sätze definieren, stellt sich die Frage nach den Ausdrucksmöglichkeiten des Symbolismus, der im Tractatus entworfen wird. Wenn man den aussagenlogischen Teil dieses Symbolismus betrachtet und die Anwendung der N-Operation auf endliche Mengen von Sätzen einschränkt, stellt sich das Problem begrenzter Ausdrucksmöglichkeiten der Notation des Tractatus nicht. Sofern die Anwendung der N-Operation auf die Satzmenge aus zwei Sätzen, die sowohl Elementarsätze als auch zusammengesetzte Sätze sein können, mit der Anwendung der UND-Form des Sheffer-Strichs ${ }^{37}$ zusammenfällt, verfügt dieser Teil der Notation über die für ein vollständiges System der Aussagenlogik notwendigen Ausdrucksmöglichkeiten, was die Resultate Posts über die Vollständigkeit des aussagenlogischen Kalküls der Principia und über seine Äquivalenz mit einem Kalkül, dessen einziger primitiver Operator der Sheffer-Strich ist, zeigen. Wittgensteins Auffassung der konstruktiven Operationen betrachtet man dennoch als problematisch. Als ernstes Problem der Logik des Tractatus wird vor allem die im Tractatus nicht ausdrücklich verbotene Möglichkeit angesehen, dass Mengen der Sätze, auf welche die N-Operation angewandt wird, unendlich sein können. Diese Möglichkeit ist vor allem deswegen fraglich, weil durch die Anwendung der N-Operation nach Wittgenstein auch quantifizierte Sätze konstruiert werden sollen. In seinem Vorwort zum Tractatus unterstreicht Russell, dass sich Wittgensteins Behandlung der allgemeinen Sätze dadurch auszeichnet, dass die Allgemeinheitsbezeichnung nur in der Be-

\footnotetext{
${ }^{36}$ Boole.Analysis, 113, 115

${ }^{37}$ Russell und Post ziehen der UND-Form die ODER-Form des Sheffer-Strichs nur aufgrund des Vorteils vor, der von Nicod unterstrichen wird und in der Sparsamkeit des Ausdrucks der Implikation besteht. J.G.P. Nicod. „A Reduction in the Number of the Primitive Propositions of Logic". In: Proceedings of the Cambridge Philosophical Society XIX, 1916-1919, 32-41, 32
} 
stimmung der Basen der N-Operation vorkommt, und sieht das in der Definition dieser Operation gegebene Konstruktionsverfahren für Sätze insbesondere dadurch begründet, dass verallgemeinerte Sätze als Konjunktionen und Disjunktionen von Elementarsätzen konstruiert werden ${ }^{38}$. Den Grund für diese Bewertung kann man in der Behauptung Wittgensteins sehen, dass als Basen der N-Operation auch sämtliche Werte einer Funktion fx auftreten können ${ }^{39}$, sowie in dem sich in dieser Auffassung realisierenden Verzicht Wittgensteins, formale Darstellungen allgemeiner Sätze als primitive Symbole zu behandeln, wie es Russell und Whitehead in den Principia Mathematica $\operatorname{tun}^{40}$. Die Russellsche Meinung ist auch heute verbreitet. So charakterisiert Wang (1992) die Auffassung Wittgensteins als Gleichsetzung eines Allsatzes mit einem logischen Produkt und eines Existenzsatzes mit einer logischen Summe ${ }^{41}$. In einem seiner späteren Manuskripte bestätigt Wittgenstein den Einwand Russells, indem er die Möglichkeit verneint, die Auffassung der Sätze der Gestalt „(x).fx“ als eines logischen Produkts und , $(\exists \mathrm{x}) . \mathrm{f} \mathrm{x}^{\prime \prime}$ als einer logischen Summe aufrechtzuerhalten, da sich das logische Produkt nicht für jeden Satz finden lässt ${ }^{42}$. Auch Anscombe (1959) weist darauf hin, dass Wittgenstein als Fehler des Tractatus die Unfähigkeit betrachtete, zwischen „dots of laziness“, die man gebraucht, um einerseits ein endliches Alphabet und andererseits eine unendliche Reihe darzustellen, zu unterscheiden ${ }^{43}$.

Sowohl das Zugeständnis Wittgensteins als auch die Auffassung Russells widerspricht der kritischen These, welche Wittgenstein im Satz 5.521 gegen Russells und Whiteheads Einführung der Allgemeinheitsbezeichnung richtet. Deshalb ist es denkbar, dass Wittgenstein, indem er die Allgemein-

\footnotetext{
${ }^{38}$ B. Russell. „Vorwort [zum Tractatus]“. In: B. McGuinness, J. Schulte (Hrsg.). L. Wittgenstein. Logisch-philosophische Abhandlung. Kritische Edition. Frankfurt am Main, Suhrkamp, 1989, 258-286, 271-272

${ }^{39}$ TLP, 5.52

${ }^{40} \mathrm{Vgl}$. PM, \$9.

${ }^{41}$ H. Wang. "What is Logic?”. In: K. Puhl (Hrsg.). Wittgenstein's Philosophy of Mathematics. Proceedings of the $15^{\text {th }}$ International Wittgenstein-Symposium 20/II, Wien, Verlag Hölder-Pichler-Tempsky, 1993, 11-23, 23

${ }^{42}$ L. Wittgenstein. „IX. Philosophische Grammatik“ (im weiteren: IX.Grammatik). In: L. Wittgenstein. Wiener Ausgabe 5. Wien, New York, Springer, 1993, 6

${ }^{43}$ Anscombe.Introduction, 134
} 
heitsbezeichnung als Argument bezeichnet ${ }^{44}$, ein solches Argument als Wertverlauf einer Funktion versteht. Ein solcher Wertverlauf wäre laut dem Satz 5.501 durch die Funktion selbst, d.h. durch ein Zuordnungsgesetz definiert, was bedeuten könnte, dass die Anwendung der N-Operation auf einen Wertverlauf keine zusammengesetzte Operation wäre, die als Konjunktion oder Disjunktion von Sätzen, welche Namen von Gegenständen enthalten, darstellbar ist.

Fogelin betrachtet 1976 in der zweiten Auflage seines Buches Wittgenstein $^{45}$ Wittgensteins Analyse der verallgemeinerten Sätze als Beleg dafür, dass die Ausdrucksmöglichkeiten der Notation des Tractatus (der Notation, deren Prinzipien Wittgenstein im Tractatus formuliert) beschränkt sind. Betrachtet man diese Notation als Notation der Prädikatenlogik der ersten Stufe, kann man nach Meinung Fogelins z.B. kein formales Äquivalent des Satzes formulieren, dem ein formaler Ausdruck der Prädikatenlogik mit einem zweistelligen Prädikat und einer gemischten Quantifikation entspricht. Diese Behauptung löst eine Diskussion aus, in deren Rahmen Geach $(1981)^{46}$ den Symbolismus Wittgensteins ergänzt, um zu zeigen, dass dieser doch den Anspruch der Notation, die für die Konstruktion einer Weltbeschreibung gebraucht werden kann, erfüllt. Notationale Vorschläge Geachs beruhen vor allem auf der Idee, dass sich eine Funktion von zwei Argumenten als eine Funktion von einem Argument darstellen lässt, wenn man den Wert eines der Argumente festhält. Eine weitere These, die der Argumentation Geachs zugrunde liegt, ist der Satz 5.501 des Tractatus, der festlegt, wie die Werte der Variablen $\xi$, welche in der Definition der NOperation die Basen dieser Operation andeutet, festgesetzt werden. Eine der drei Möglichkeiten der Festsetzung besteht in der „Angabe einer Funktion $\mathrm{fx}$, deren Werte für alle Werte von $\mathrm{x}$ die zu beschreibenden Sätze sind $^{\text {“47 }}$. Ich betrachte Geachs Ansatz als richtig und sehe die Möglichkeit

\footnotetext{
${ }^{44}$ TLP, 5.523

${ }^{45}$ Fogelin.Wittgenstein, 70-71

${ }^{46}$ S. P.T. Geach. "Wittgenstein's Operator N“. In: Analysis 41, 1981, 168-171, P.T. Geach. „More on Wittgenstein’s Operator N“. In: Analysis 42, 1982, 127-128, R.J. Fogelin. „Wittgenstein's Operator N“. In: Analysis 42, 1982, 124-127. An der Diskussion beteiligte sich auch Soames, der die Meinung vertrat, welche der Meinung Geachs nahe kam. S. S. Soames. "Generality, Truth Functions, and Expressive Capacity in the Tractatus". In: The Philosophical Review XCII, 1983, 573-589.

${ }^{47}$ TLP, 5.501
} 
einer alternativen notationalen Darstellung für Sätze mit gemischter Quantifikation. Die Alternative, die ich vorschlage, beruht auf der Fregeschen Bezeichnung der Wertverläufe.

Ich benutze Buchstaben , $\mathrm{x}^{\prime \prime}$ und „, $\mathrm{y}^{\text {“ }}$ als freie Variablen, welche Gegenstände andeuten, ,a“ und „e “ als gebundene Variablen, „, $\alpha$ “ und , , $\varepsilon^{\text {“ }}$ als Variablen, die in den Aussagen über Wertverläufe ( $\alpha$ und $\varepsilon$ ) von Funktionen verwendet werden, „A“ als Namen. Den doppelten Wertverlauf einer Funktion $\Psi(\mathrm{x}, \mathrm{y})$ bezeichne ich folglich durch den Ausdruck , $\alpha \dot{\varepsilon} \Psi(\alpha, \varepsilon)^{\text {““. }}$. Man betrachte nun den Wertverlauf der Funktion $\Psi(\mathrm{x}, \mathrm{y})$ für einen bestimmten Wert der Variablen x $\dot{\varepsilon} \Psi(\mathrm{A}, \varepsilon)$. Wenn dieser Wertverlauf als Basis der Anwendung der N-Operation auftritt, ist das Resultat dieser Anwendung folgendermaßen in der Standardnotation für Quantifikation, die von Wittgenstein für seine Definition im Satz 5.52 benutzt wird, definiert:

$\mathrm{N}(\hat{\varepsilon} \Psi(\mathrm{A}, \varepsilon))=\sim(\exists \mathbf{e}) . \Psi(\mathrm{A}, \mathbf{e})$.

Die Anwendung der N-Operation auf diesen Satz ergibt einen Existenzsatz $\mathrm{N}(\mathrm{N}(\dot{\varepsilon} \Psi(\mathrm{A}, \varepsilon)))=(\exists \mathbf{e}) . \Psi(\mathrm{A}, \mathbf{e})$.

Das Resultat der Anwendung der N-Operation auf den Wertverlauf $\varepsilon ́ \mathrm{~N}(\Psi(\mathrm{A}, \varepsilon))$ ist ein Allsatz

$\mathrm{N}(\dot{\varepsilon} \mathrm{N}(\Psi(\mathrm{A}, \varepsilon)))=(\forall \mathbf{e}) . \Psi(\mathrm{A}, \mathbf{e})$.

Die Sätze, die auf diese Weise gewonnen werden, können als Ausdrücke von Funktionen betrachtet werden, wenn der Name „A“, der in ihnen vorkommt, durch die Variable x ersetzt wird. Deshalb kann man von den Wertverläufen dieser beiden Funktionen $\alpha \mathrm{N}(\mathrm{N}(\dot{\varepsilon} \Psi(\alpha, \varepsilon)))$ und $\alpha ́ \mathrm{~N}(\varepsilon \dot{\mathrm{N}}(\Psi(\alpha, \varepsilon)))$ sowie von den Wertverläufen der Funktionen, deren Werte die Werte der durch die gewonnenen Sätze bestimmten Funktionen verneinen, $\alpha \mathrm{N}\left(\mathrm{N}\left(\mathrm{N}\left(\hat{\varepsilon}^{\prime} \Psi(\alpha, \varepsilon)\right)\right)\right)$ und $\alpha \mathrm{N}(\mathrm{N}(\dot{\varepsilon} \mathrm{N}(\Psi(\alpha, \varepsilon))))$ reden und sie als Basen der N-Operation betrachten. Durch die Anwendung der N-Operation auf diese Wertverläufe gewinnt man folgende Sätze:

$\mathrm{N}(\mathrm{N}(\alpha \dot{N}(\mathrm{~N}(\dot{\varepsilon} \Psi(\alpha, \varepsilon)))))=(\exists \mathbf{a}):(\exists \mathbf{e}) \cdot \Psi(\mathbf{a}, \mathbf{e})$

$\mathrm{N}(\mathrm{N}(\alpha \dot{N}(\varepsilon ́ \mathrm{~N}(\Psi(\alpha, \varepsilon)))))=(\exists \mathbf{a}):(\forall \mathbf{e}) \cdot \Psi(\mathbf{a}, \mathbf{e})$

$\mathrm{N}(\alpha \dot{N}(\mathrm{~N}(\mathrm{~N}(\dot{\varepsilon} \Psi(\alpha, \varepsilon)))))=(\forall \mathbf{a}):(\exists \mathbf{e}) \cdot \Psi(\mathbf{a}, \mathbf{e})$

$\mathrm{N}(\alpha \dot{N}(\mathrm{~N}(\dot{\varepsilon} \mathrm{N}(\Psi(\alpha, \varepsilon)))))=(\forall \mathbf{a}):(\forall \mathbf{e}) . \Psi(\mathbf{a}, \mathbf{e})$.

Im Zusammenhang mit der Problematik solcher Darstellungen stellt sich die Frage, warum Wittgenstein darauf verzichtet, die Quantifizierung als eine selbständige logische Operation zu betrachten. Die Tagebücher zei- 
gen, dass die Quantifizierung für ihn anfangs eine logische Operation neben der Strich-Operation, d.h. einer Wahrheitsoperation, ist ${ }^{48}$. Im Tractatus behauptet er aber, dass er „den Begriff Alle von der Wahrheitsfunktion“ trennt ${ }^{49}$.

Die Quantifizierung ist eine zusammengesetzte Operation, sofern sie, auf den Ausdruck einer propositionalen Funktion angewandt, in der Anwendung der Operation der Substitution besteht. Diese letztere Operation ist ihrerseits komplex, worauf 1929 Curry hinweist, wenn er Substitution als Einsetzen von Konstanten für Variablen in dem Ausdruck einer propositionalen Funktion definiert und sie als eine zusammengesetzte und strukturierte Operation betrachtet ${ }^{50}$. In den Tagebüchern geht Wittgenstein davon aus, dass alle Operationen ,aus den Grundoperationen zusammengesetzt“ $\operatorname{sind}^{51}$ und dass völlig allgemeine Sätze durch einen „graduelle[n] Übergang vom elementaren Satz ${ }^{\text {c52 }}$ gewonnen werden können. Die Operation der Substitution sollte ihm vor allem aus den Fregeschen Grundgesetzen bekannt sein, wo ihre Regeln als Regeln des Ersatzes von Buchstaben explizit formuliert sind ${ }^{53}$. Im Tractatus setzt Wittgenstein den Gebrauch der Operation der Ersetzung von Namen durch Variablen voraus, wenn er im Satz 3.315 über das Gewinnen des Ausdrucks für ein logisches Urbild (eine logische Form) spricht. Diese Operation ist aber nach der Definition Wittgensteins keine logische Operation, sofern ihr Resultat kein Satz, sondern eine in seiner Terminologie Satzvariable und mithin eine Bezeichnung eines formalen Begriffs ist. Ähnliche Problematik beschäftigt Wittgenstein in den Tagebüchern, wo er sich fragt, was die Basis der Quantifizierung sei ${ }^{54}$. Wenn diese Basis ein bereits mit Hilfe einer Variablen verallgemeinerter Satz sein sollte, ist diese Basis kein Satz. Sollte diese Basis ein Elementarsatz sein, enthält die Anwendung der Operation der Quantifizierung die Operation der Ersetzung von Namen und anderen konstanten Ausdrücken durch Variablen, welche insofern keine logische Operation im

\footnotetext{
48 Tagebücher, 13.7.16

${ }^{49}$ TLP, 5.521

${ }^{50}$ Curry.Substitution, 368-369

${ }^{51}$ Tagebücher, 26.11.16

${ }^{52}$ Ebd., 14.10.14

${ }^{53}$ GGA I, \$48

${ }^{54}$ Tagebücher, 11.5.16
} 
Sinne Wittgensteins ist, als sie sowohl auf Sätze als auch auf Namen, die keine Struktur haben, angewandt wird.

Es besteht allerdings die Möglichkeit, die Quantifizierung als eine bejahende Operation aufzufassen. Die Anerkennung dieser Möglichkeit bedeutet einen Verzicht auf die Identifizierung der Anwendung der N-Operation mit dem Zweck des Gewinnens allgemeiner Sätze mit der Konjunktion oder Disjunktion von Elementarsätzen. Sofern ein verallgemeinerter Satz durch eine einfache Anwendung der N-Operation konstruierbar ist, ist der Zusammenhang zwischen einem Elementarsatz und einem allgemeinen Satz kein konstruktiver Zusammenhang, sondern einer zwischen der Prämisse und dem Schluss eines logischen Schlusses. Diese Möglichkeit fände auch eine Entsprechung unter den Fregeschen Regeln in der Regel der Verwandlung eines lateinischen Buchstaben in einen deutschen ${ }^{55}$.

Die Wittgensteinsche Auffassung der Allgemeinheit weist Ähnlichkeiten mit der Husserlschen Auffassung der Allgemeinheit auf. Im zweiten Band der Logischen Untersuchungen unterscheidet Husserl zwischen drei Arten der Allgemeinheit. Die erste Art der Allgemeinheit ist die Allgemeinheit des Prädikats, die zu seiner logischen Funktion gehört und mit der Allgemeinheit der Russellschen propositionalen Funktion oder des Fregeschen Begriffs gleichgesetzt werden kann. Sie besteht darin, dass jedes Prädikat eine Menge von Sätzen definiert. Eine andere Art der Allgemeinheit ist die Allgemeinheit einer Spezies, welche Husserl als Allgemeinheit der Bedeutung ansieht. Die dritte Art der Allgemeinheit ist die Allgemeinheit, die als Bezugspunkt der Prädikation in einem allgemeinen Satz auftritt. Das Meinen dieser Allgemeinheit, die als Allgemeinheit eines Begriffsumfangs verstanden werden kann, kann nicht auf das Meinen der einzelnen Glieder des Umfangs reduziert werden ${ }^{56}$. Fasst man den Begriffsumfang als Wertverlauf einer Funktion, deren Werte Sätze einer bestimmten Form sind, und das Fundiert-sein der Prädikation in der gemeinten Allgemeinheit korrelativ als Anwendung einer Operation auf, hat man die Wittgensteinsche Auffassung der Konstruktion der allgemeinen Sätze mittels N-Operation.

${ }^{55}$ GGA I, $\S \S 17,48$

${ }^{56}$ E. Husserl. Logische Untersuchungen (im weiteren: LU). Zweiter Band. I und II

Teil. Hrsg. von U. Panzer, The Hague/Boston/Lancaster, 1984 (Husserliana XIX/1, 2), $152-153$ 
$\S 1 \mathrm{c}$. Wittgensteins bejahende Operationen

Im Tractatus sowie in früheren Texten analysiert Wittgenstein nicht nur konstruktive Operationen, sondern auch bejahende und verneinende Operationen. Seiner Ausdrucksweise folgend, kann man von Bejahung und Verneinung ${ }^{57}$, sowie von verneinenden und verneinten Sätzen ${ }^{58}$ sprechen, wie auch behaupten, dass ein Satz einen anderen bejaht oder verneint ${ }^{59}$. Diese drei Begriffspaare geben Anlass zur Unterscheidung zwischen zwei Arten der Gegenüberstellung. Als Bejahung kann man einen Satz auffassen, dessen formale Darstellung in der traditionellen aussagenlogischen Notation kein Verneinungszeichen als Hauptzeichen enthält und folglich mit keinem oder mit einer geraden Anzahl von Verneinungszeichen beginnt. Die Verneinung ist ein Satz, dessen formale Darstellung ein Verneinungszeichen als Hauptzeichen enthält und folglich mit einem Verneinungszeichen beginnt, so dass die Anzahl der diesem Zeichen unmittelbar folgenden Verneinungszeichen 0 oder gerade ist. Die so verstandenen Bejahung und Verneinung sind Sätze einer bestimmten Gestalt. Für jede Bejahung gibt es eine ihr entsprechende Verneinung und umgekehrt, so dass einer, wenn ihm ein Satz gegeben ist, sein Korrelat angeben kann. Eine Verneinung und die ihr entsprechende Bejahung kann man als verneinenden und verneinten Satz bezeichnen und ihre logische Beziehung durch das Gesetz der doppelten Negation charakterisieren. So aufgefasst, sind Bejahung und Verneinung Resultate bestimmter konstruktiver Operationen, sofern die Verneinung eine Wahrheitsfunktion der ihr entsprechenden Bejahung ist. Man kann einen gegebenen Satz (Bejahung oder Verneinung) auch als Resultat der Operation des logischen Schließens betrachten, deren Basis die Verneinung des Korrelats des gegebenen Satzes ist.

Von dieser Art der Gegenüberstellung kann man diejenige unterscheiden, die in der Charakterisierung der Bejahung und Verneinung als Operationen von Sätzen auf anderen Sätzen zum Ausdruck kommt. Dass ein Satz „r“ einen anderen Satz , "s“ verneint, bedeutet laut dem Tractatus, dass r s widerspricht $^{60}$, und kann somit durch die Feststellung ausgedrückt werden,

\footnotetext{
${ }^{57}$ TLP, 4.064, 5.44, 6.231

58 Ebd., 4.0641

${ }^{59}$ Ebd., 5.124-5.1241, 5.513-5.514, 5.44

${ }^{60}$ Ebd., 5.12
} 
dass die Konjunktion von $\mathrm{r}$ und $\mathrm{s}$ (der Satz „r.s“) eine Kontradiktion ist. Dass ein Satz „r“ $\mathrm{r}^{\text {“ }}$ einen Satz , ,“ bejaht, bedeutet, dass s aus $\mathrm{r}$ folgt ${ }^{61}$ oder dass der Satz „r $\supset s^{\text {“ }}$ eine Tautologie ist. Der tautologische Charakter der Implikation, deren Antezedens die Prämisse ${ }^{62}$ und Konsequens der Schlusssatz eines Schlusses ist, wird als Bedingung des logischen Schließens in dem Begriff der Wahrheitsgründe des Satzes und dem Begriff der Wahrscheinlichkeit, welche die Prämisse dem Schlusssatz gibt, festgelegt. Beide Begriffe charakterisieren Folgebeziehungen zwischen Sätzen. Betrachtet man als Beispiel die Konjunktion von zwei Sätzen ,p.q“ und den aus diesem Satz folgenden Satz ,p“, die man der Bezeichnungsweise Wittgensteins entsprechend als „(WFFF)(p,q)“ und „(WFWF)(p,q)“ niederschreiben kann, dann ist die Anzahl der Wahrheitsgründe der Konjunktion $1\left(\mathrm{~W}_{\mathrm{p} . \mathrm{q}}=1\right)$. Die Anzahl der Wahrheitsgründe des Satzes ,p", die zugleich die Wahrheitsgründe der Konjunktion sind, ist auch $1\left(\mathrm{~W}_{\mathrm{p} . \mathrm{q}, \mathrm{p}}=1\right)$. Das Maß der Wahrscheinlichkeit, die der Satz „p.q“ dem Satz „p“ gibt, ist $\mathrm{W}_{\mathrm{p} . \mathrm{q}, \mathrm{p}}: \mathrm{W}_{\mathrm{p} . \mathrm{q}}=1$, was bedeutet, dass die Wahrheitsgründe von p.q alle in den Wahrheitsgründen von $\mathrm{p}$ enthalten sind. Somit folgt $\mathrm{p}$ aus der Konjunktion ,p.q“63. Auf eine dieser Definition der Beziehung zwischen den Wahrheitsgründen der Prämisse und des Schlusssatzes eines Schlusses ähnliche Weise werden in einigen Theorien der formalen Deduktion die Bedingungen charakterisiert, unter welchen man die Figur p.q

$\mathrm{p}$

als eine logische Deduktionsregel definieren und von $\mathrm{p}$ behaupten kann, dass $p$ aus p.q logisch folgt. Eine Figur der Gestalt

$A_{1}, A_{2}, \ldots, A_{n}$

$\mathrm{C}$

ist eine logische Deduktionsregel und $\mathrm{C}$ folgt logisch aus den Prämissen $A_{1}, A_{2}, \ldots, A_{n}$, wenn der Ausdruck der Gestalt „, $\left(A_{1} .\left(A_{2} \ldots . . A_{n}\right) \ldots\right) \supset C^{c}$ eine

\footnotetext{
${ }^{61}$ Ebd., 5.124

${ }^{62}$ Die Konjunktion aller Prämissen eines Schlusses kann man als seine einzige Prämisse definieren.

${ }^{63}$ TLP, 5.15-5.152
} 
Tautologie ist und somit die Bedingung erfüllt, dass $\mathrm{C}$ nicht falsch sein kann, wenn die Prämissen $A_{1}, A_{2}, \ldots, A_{n}$ wahr sind. Diese Definition erlaubt es insbesondere, von der Formulierung logischer Prinzipien in Gestalt von logischen Gesetzen zur Formulierung dieser Prinzipien in Gestalt von logischen Schlussregeln überzugehen ${ }^{64}$. Die Anerkennung dieser Übergangsmöglichkeit charakterisiert auch Wittgensteins Vorstellung von der Rolle der logischen Sätze im Symbolismus.

Zur Problematik der Wittgensteinschen Charakterisierung bejahender und verneinender Operationen kann man folgende Fragestellungen zählen:

1. Was kann man als Zeichen der Operationen des logischen Schließens betrachten?

2. Welches sind die Beziehungen zwischen bejahenden und verneinenden Operationen?

3. Welches sind die Beziehungen zwischen den Operationen des logischen Schließens und den konstruktiven Operationen?

\footnotetext{
${ }^{64}$ S. z.B. O.F. Serebrjannikov. Heuristische Prinzipien und logische Kalküle (im weiteren: Serebrjannikov.Heuristische Prinzipien). München, Salzburg, Wilhelm Fink Verlag, 1974, 122-123, 126. Den Vorteil dieser Definition im Vergleich zu anderen in der modernen Theorie der Folgebeziehungen gängigen Definitionen sehe ich darin, dass sie die Beziehungen der Wahrheitsbedingungen von Prämissen und Schluss berücksichtigt. Für gewöhnlich wird in einer Definition der logischen Folgebeziehung der strukturelle Zusammenhang zwischen Prämissen und Schluss und die Gültigkeit der logischen Regeln verlangt. Wegen der Möglichkeit verschiedener Einschränkungen auf die Gestalt der Sätze, die aus Prämissen folgen können und die ihre Wurzeln in den intuitiven Vorstellungen von Folgebeziehungen haben, kann man im allgemeinen nicht behaupten, dass der tautologische Charakter einer Implikation bedeutet, dass ihr Konsequens aus ihrem Antezedens logisch folgt. S. A.A. Zinov’ev. „Die logische und die physische Folgebeziehung“ (im weiteren: Zinov'ev.Folgebeziehung). In: G. Kröber (Hrsg.). Studien zur Logik der wissenschaftlichen Erkenntnis. Berlin, Akademie Verlag, 1967, 113-190, 153. Sofern die Theorie Wittgensteins als Theorie angesehen werden kann, die der klassischen von Russell und Whitehead formulierten Theorie der Folgebeziehung (zum Begriff dieser Theorie s. A.A. Sinowjew. Komplexe Logik. Grundlagen einer logischen Theorie des Wissens (im weiteren: Sinowjew.Komplexe Logik). Braunschweig, Vieweg, Basel, C.F. Winter'sche Verlagshandlung, 1970, 97) entspricht und deren einzige einschränkende Forderung nicht in dem Ausschließen irgendwelcher Sätze der Aussagenlogik als Formulierungen von möglichen Schlussregeln besteht, sondern darin, dass als Prämissen und Folgen nur sinnvolle Sätze auftreten können, kann man von der Problematik der angesprochenen intuitionsbedingten Einschränkungen absehen.
} 
ad 1. Die Behauptung Wittgensteins, es gäbe für jeden Satz nur einen ihn verneinenden Satz ${ }^{65}$, sowie die Definierbarkeit des verneinenden Satzes durch den Widerspruch, welchen die Konjunktion dieses Satzes mit dem von ihm verneinten Satz ergibt, lassen anfangs vermuten, dass als Operator der verneinenden Operation der Verneinungs-Operator auftritt. Dagegen spricht erstens das Fehlen eines entsprechenden Bejahungs-Operators und zweitens der Umstand, dass nach Wittgenstein Sätze selbst auf anderen, den zu bejahenden sowie zu verneinenden Sätzen operieren ${ }^{66}$. Das Fregesche Behauptungszeichen, das die Rolle eines Bejahungs-Operators beanspruchen könnte, erfüllt keine mit dem Verneinungs-Operator vergleichbare Funktion in dem Symbolismus. Will man es als einen Operator auffassen und zugleich bei der Fregeschen Charakterisierung dieses Zeichens bleiben, muss man das aus dem Behauptungszeichen und einem Satz „p“ bestehende Symbol als ein Äquivalent des Satzes ,,p ist ein logischer Satz“ betrachten, der für Wittgenstein gleichbedeutend mit dem Satz ,p ist eine Tautologie" ist. Dann aber muss man das Symbol, in welchem das Verneinungszeichen vor $\mathrm{p}$ vorkommt, als den Satz ,p ist keine Tautologie“ lesen können. Dieser Satz ist jedoch mit dem Satz „, p“ nicht äquivalent. Ist $p$ ein sinnvoller Satz, dann ist der Satz ,p ist keine Tautologie“ wahr unabhängig davon, ob $\mathrm{p}$ wahr oder falsch ist, während die Verneinung von $\mathrm{p}$ (der Satz , $\sim p^{\text {") }}$ sowohl wahr als auch falsch sein kann.

Warum Wittgenstein keinen besonderen Operator für die Operationen des logischen Schließens einführt, könnten einige moderne Entwicklungen der allgemeinen Theorie der Folgebeziehungen zeigen, die solche Operatoren benutzen: Sätze, welche besondere Zeichen für Folgebeziehungen enthalten, gehören der Sprache einer höheren semantischen Stufe an als die Sätze, die in Hinsicht auf das Bestehen zwischen ihnen einer Folgebeziehung untersucht werden. In den auf diese Weise aufgefassten Sätzen über Folgebeziehungen tritt der Operator als Zeichen einer logischen Beziehung auf ${ }^{67}$. Diese Betrachtungsweise ist mit dem Verzicht Wittgensteins, eine Aussage über logische Beziehungen als sinnvoll anzusehen ${ }^{68}$, kaum vereinbar.

\footnotetext{
${ }^{65}$ TLP, 5.513

${ }^{66}$ Ebd., 5.124

67 Zinov'ev.Folgebeziehung, 117

${ }^{68}$ TLP, 4.1241
} 
Laut Wittgenstein kann man das, was aus einem Satz folgen kann, an dem Zeichen des Satzes selbst, insbesondere an seiner Struktur, ablesen ${ }^{69}$. Diese Behauptung könnte bedeuten, dass Wittgenstein die Einführung besonderer, von den Sätzen verschiedener Symbole für Folgebeziehungen für überflüssig hält. Wenn die Bejahung sowie die Verneinung als interne Beziehungen zwischen Sätzen betrachtet werden ${ }^{70}$, lassen sich diese Beziehungen dadurch ausdrücken, dass ein Satz als Resultat einer Operation dargestellt wird, die ihn aus anderen Sätzen hervorbringt ${ }^{71}$. Die Basis der Operation der Bejahung ist ein bejahender Satz und ihr Resultat ein bejahte Satz, d.h. einer der Sätze, die aus dem bejahenden Satz logisch folgen. Die Basis der Verneinung ist ein verneinender Satz und ihr Resultat der verneinte Satz, d.h. der Satz, der dem verneinenden widerspricht. Als Operationssymbole fungieren die Vorschriften zur Ausführung von bejahenden und verneinenden Operationen, die im Fall der Bejahung in Gestalt von tautologischen Implikationen angegeben werden können. Im Fall der Verneinung hat die Vorschrift die Gestalt einer kontradiktorischen Konjunktion. Dass Wittgenstein Sätze selbst, d.h. auch darstellende Symbole, als Operationssymbole auffasst, ist insofern nicht ungewöhnlich, als seine Auffassung z.B. mit der Booleschen Betrachtung von Variablen für Auswahlsymbole als Operationssymbolen übereinstimmt.

Dass Wittgenstein die verneinenden Operationen den bejahenden gegenüberstellt und gleichzeitig die Verneinung als eine konstruktive Operation behandelt, lässt sich darüber hinaus durch die Möglichkeit solcher verschiedener Auffassungen der Verneinung wie Unbeweisbarkeit, Widerlegbarkeit und Absurdität bei der Konstruktion formaler Systeme erklären. Curry weist zum Teil in Anlehnung an Carnap darauf hin, dass zum Bestand einer formalen Theorie außer beweisbaren Behauptungen (Theoremen) auch widerlegbare Behauptungen gehören können ${ }^{72}$. Die Menge solcher Behauptungen kann wie die Menge von Theoremen induktiv auf der Basis von Gegenaxiomen (counteraxioms) definiert werden. Carnap betrachtet als Gegenaxiome die Regeln der Widerlegung, welche die direkt

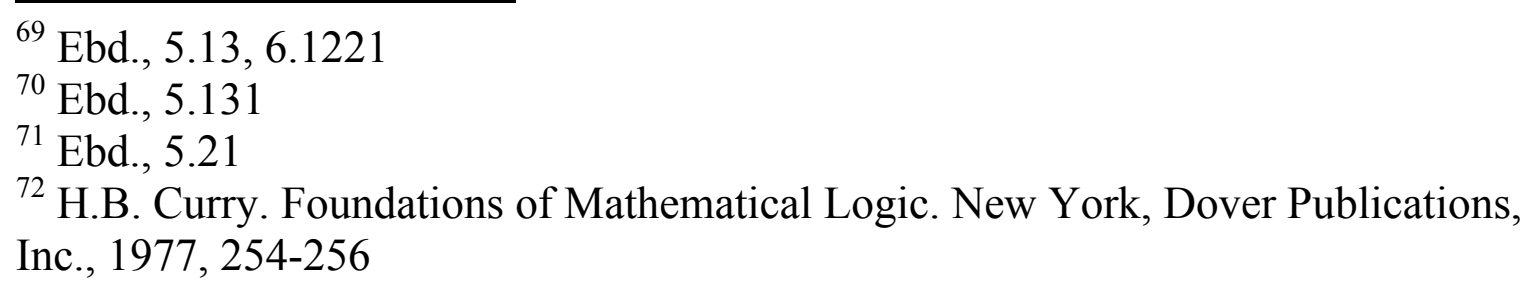


widerlegbaren Ausdrücke eines Kalküls angeben ${ }^{73}$. Eine solche Betrachtungsweise erlaubt es, die Widerlegbarkeit einer Behauptung als Beweisbarkeit ihrer Verneinung aufzufassen, was der Verneinung den gleichen Status wie anderen logischen konstruktiven Operationen wie Konjunktion oder Disjunktion verleiht. Sofern Wittgenstein die Möglichkeit zulässt, das logische Schließen auch durch Kontradiktionen zu beschreiben ${ }^{74}$, scheint

${ }^{73}$ R. Carnap. Introduction to Semantics. Cambridge, Massachusetts, Harvard University Press, 1968, 162-165

${ }^{74} \mathrm{~S}$. TLP, 6.1202. Wenn man nach einem Beispiel für diese Möglichkeit sucht, könnte man ein solches in der Verneinung der Tautologie „p.q: $\supset$.p“ sehen. Dass die Sätze „p.q“ und „p“, zum Satz „, (p.q: $\supset$.p)“ verbunden, eine Kontradiktion ergeben, heißt, dass die Konjunktion des Satzes ,p.q“ und der Verneinung von p eine Kontradiktion ist. Diese Behauptung lässt sich ihrerseits als Feststellung deuten, dass es unmöglich ist, dass p aus p.q logisch nicht folgt. Diese Deutung schlägt 1927 Chadwick vor, der die Voraussetzung der Definition des Begriffs des logischen Folgens in der Einführung des Begriffs der logischen Unmöglichkeit sieht. Obwohl Chadwicks Auffassung von Fakten und ihre Analyse in Entitäten und ihre Eigenschaften oder Beziehungen auf eine de re Auffassung von Modalitäten hinausläuft, sind für ihn die logischen Modalitäten, welche die Folgebeziehungen zwischen Sätzen charakterisieren, Modalitäten de dicto, sofern sie Sätze charakterisieren. Chadwick sieht in Wittgensteins Begriff „eine Tautologie zu sein“ (,,being a tautology") die Definition eines modalen Begriffs der zweiten Art, nämlich des Begriffs ,logisch notwendig zu sein“ (,,being logically necessary“) (J.A. Chadwick. „Logical Constants”. In: Mind 36, 1927, 1-11, 7). 1982 weist von Wright darauf hin, dass die Modalitätsauffassung, welche die modalen Begriffe durch nicht-modale Begriffe „definiert“, reduktionistisch ist, wie die Auffassung Carnaps. Einer Auslegung des Tractatus, die zu Definitionen der Notwendigkeit und der Unmöglichkeit durch wahrheitsfunktionale Begriffe führt, wirft von Wright vor, dass sie den Begriff der Gewissheit, der für Wittgenstein einen epistemischen und in diesem Sinn intensionalen Charakter hat, mit dem Begriff der Notwendigkeit identifiziert. Er schlägt vor, den Begriff der Sinnhaftigkeit des Satzes (dass der sinnvolle Satz wahr oder falsch sein kann) als einen undefinierbaren Begriff zu behandeln, der mit dem modalen Begriff der Kontingenz (bezeichnet durch den Operator „C“) gleichgesetzt werden kann (von Wright.Modal Logic, 188, 192, 195-197, 200). Zur Begründung dieser Gleichsetzung s. auch A. Maury. The Concept of Sinn and Gegenstand in Wittgenstein's Tractatus. In: Acta Philosophica Fennica XXIX, 1977, 24-29. Die Begriffe der Notwendigkeit und Unmöglichkeit sind nach von Wright mit Hilfe des Begriffs der Kontingenz im Sinne der Sinnhaftigkeit definierbar, so dass man aussagenlogische Tautologien und Kontradiktionen mit Hilfe des Begriffs der Kontingenz als solche charakterisieren und ihnen einen bestimmten modalen Status zuschreiben kann. Die von von Wright vorgeschlagene Rekonstruktion der Logik des Tractatus als eines Systems der modalen Logik, das mit dem System S5 deduktiv äquivalent ist, hebt den rationalistischen Charakter dieser Logik als Modallogik von modalen Aussagen hervor 
es, dass das Gegenüberstellen von verneinenden und bejahenden Operationen einerseits und die Möglichkeit, die verneinenden Operationen mit Hilfe des Verneinungszeichens auszudrücken, andererseits auf die Auffassung der Verneinung als Widerlegbarkeit hindeuten könnte.

ad 2. Ein weiteres Problem der Wittgensteinschen Auffassung der Operationen besteht darin, dass das gegenseitige Verhältnis zwischen bejahenden und verneinenden Operationen im Tractatus nicht definiert ist. Die Behauptung Wittgensteins, dass die Eigenschaft der Bejahung darin besteht, dass man sie als doppelte Verneinung auffassen kann $^{75}$, kann eine solche Definition kaum ersetzen. Der Grund dafür ist nicht die viel beklagte Mehrdeutigkeit der Terminologie Wittgensteins ${ }^{76}$, sondern die Tatsache, dass diese Behauptung mittels einer Tautologie die Basis und das Resultat einer bestimmten unter mehreren anderen bejahenden Operationen angibt. Dass man in der zweiwertigen Aussagenlogik den Gegensatz zwischen einer bejahenden und einer ihr entsprechenden verneinenden Aussage durch die Begriffe der Wahrheit und Falschheit beschreibt, sowie die Sprechweise Wittgensteins, der das Ausführen der beiden Operationen den Sätzen delegiert, scheint darauf hinzuweisen, dass diese Operationen komplementär zueinander sind.

Dies ist aber, wenn die Verneinung mit einer konstruktiven Operation gleichgesetzt wird, nicht der Fall. Aufschlussreich ist in dieser Hinsicht die Unterscheidung, die Frege 1919 analysiert. Frege zieht die Möglichkeit in Betracht, Bejahung und Verneinung als Eigenschaften der Tat des Urteilens zu fassen, und kommt zum Schluss, dass die Verneinung zum Gedanken, über welchen das Urteil gefällt wird, gehört und kein Bestandteil oder Charakteristikum des Urteilens ist ${ }^{77}$. Diese Fregesche Analyse könnte erklären, warum Wittgenstein die Fähigkeit des Bejahens den Sätzen zuschreibt. Zunächst entgeht er dadurch der Notwendigkeit, zwischen dem Urteilen als Handlung und dem Gedanken als dem Gegenstand einer solchen Handlung zu unterscheiden und somit den Gedanken als Sinn des Satzes anzuerkennen. Außerdem erhält er ein zusätzliches Argument gegen

und enthält die aussagenlogischen Tautologien als ein Subsystem (vgl. von

Wright.Modal Logic, 193, 197, 200).

${ }^{75}$ TLP, 6.231

${ }^{76}$ S. z.B. Sundholm.Form, 60.

${ }^{77}$ Verneinung, 74-75 
die Einführung des Behauptungszeichens in den logischen Symbolismus: Ein logischer Satz zeigt sich nicht nur durch sein eigenes Satzzeichen, sondern auch dadurch, dass er das Bejahen sinnvoller Sätze bestimmter Gestalt ermöglicht. Deswegen bedarf ein logischer Satz keines syntaktischen „Anhängsels“ wie des Fregeschen Behauptungszeichens. Aber auch bei Wittgenstein ist die Verneinung kein Gegenpol zur Bejahung. Zunächst ist das Resultat der Verneinung für jede Basis dieser Operation eindeutig bestimmt $^{78}$, was für Resultate der Bejahung eines gegebenen Satzes nicht der Fall ist, sofern ein Satz mehrere Sätze wie die Konjunktion ihre beiden Konjunkte bejahen kann. Des weiteren hat die Bejahung keinen Repräsentanten in dem Symbolismus außer einer Tautologie, der es erlauben könnte, die Beziehungen zwischen Bejahung und Verneinung in der Form einer Gleichung oder einer Äquivalenz auszudrücken: Sofern Wittgenstein keine metasprachlichen Formalisierungen benutzt, muss eine solche Beziehung für jede Tautologie, d.h. für jede Menge tautologischer Sätze gleicher Struktur, definiert werden. Durch die Anwendung der konstruktiven Operation der Verneinung kann man jedoch einen Satz gewinnen, welcher der Basis der Verneinung entgegengesetzt ist. Ein solches Gewinnen zum Zwecke des Beweises eines Satzes wäre gleichbedeutend mit einem Schritt eines indirekten Beweises. Dann könnte die Beziehung zwischen bejahenden und verneinenden Operationen durch ihre Rolle in Beweisen definiert werden.

Als einen Kandidaten für die Rolle der zu der bejahenden inversen Operation kann man die dem Verfahren der Beweissuche für einen Satz entsprechende Suche nach den Prämissen für das Resultat der Bejahung betrachten. Eine solche Suche wird als eine der heuristischen Aufgaben eines Sequenzenkalküls angesehen. Die Besonderheit des Sequenzenkalküls besteht darin, dass seine Regeln zugleich als die ein Lösungsschema bestimmende Analyse- und als die das Lösungsschema in eine wirkliche Lösung verwandelnde Synthese- Prinzipien funktionieren ${ }^{79}$. Die Lösbarkeit der Aufgabe der Beweissuche für Sequenzenkalküle ohne Schnitt (ohne Strukturregeln) wird durch die Teilformeln-Eigenschaft der Beweise (der Herleitungen) begründet. Gentzen definiert als Teilformeln-Eigenschaft der Her-

\footnotetext{
${ }^{78}$ TLP, 5.513

${ }^{79}$ Serebrjannikov.Heuristische Prinzipien, 26-27, 34-35, 43, 56-57
} 
leitungen in einem Sequenzenkalkül die Eigenschaft der durch Herleitungen dargestellten Beweise, keine Umwege zu machen und nur Begriffe zu enthalten, die auch im Endergebnis vorkommen ${ }^{80}$. Dass Formeln, die in Prämissen (in den oberen Sequenzen einer Herleitung) vorkommen, Teilformeln der Formeln des Schlusses (der unteren Sequenzen derselben Herleitung) sind, erlaubt es, an Hand der unteren Sequenzen die oberen Sequenzen $\mathrm{zu}$ bestimmen. In der Behauptung über die TeilformelnEigenschaft der Beweise kann man eine Parallele zu der These Wittgensteins über den strukturbedingten Charakter des logischen Schließens ${ }^{81}$ sehen. Betrachtet man als die zu Operationen des Bejahens (oder zum Übergang von einem gegebenen Satz zu seinen logischen Folgen) inversen Operationen die Operationen der Bestimmung der bejahenden Sätze (den Übergang von einem Satz zu seinen logischen Prämissen oder zu seinem Beweis), dann definieren die logischen Schlussregeln sowohl die direkten bejahenden als auch die zu diesen inversen Operationen.

Die Definition inverser Operationen kann jedoch als entbehrlich angesehen werden. So bemerkt Lewis, dass selbst für solche Operationen wie logische Addition und logische Multiplikation die Definition der Inversen zu derartigen Problemen führt, dass sie sich in einer Algebra der Logik der BooleSchröderschen Art kaum lohnt ${ }^{82}$.

ad 3. Trotz des Umstandes, dass man sowohl bejahende als auch konstruktive Operationen als Operationen der Transformation (der Gestaltveränderung oder der Überführung) ${ }^{83}$ bestimmter gegebener Sätze in andere Sätze betrachten kann, gibt es Unterschiede, welche die Trennung der Operationenarten rechtfertigen. Während für das Ausführen der konstruktiven Operationen der Wahrheitswert ihrer Basen ohne Bedeutung ist und es nur wichtig ist, dass die Basen einen Wahrheitswert haben, d.h. Sätze sind, wird das Ausführen der Operationen des logischen Schließens nur durch die Anerkennung eines Satzes als wahren gerechtfertigt. Während konstruktive Operationen auf beliebige Sätze anwendbar sind und Sätze aller

\footnotetext{
${ }^{80}$ G. Gentzen. „Untersuchungen über das logische Schließen“ (im weiteren: Gentzen.Untersuchungen). In: Mathematische Zeitschrift 39, 1934/35, 176-210, 405-43, 195-196

${ }^{81}$ TLP, 6.12-6.1201

${ }^{82}$ C.I. Lewis. A Survey of a Symbolic Logic (im weiteren: Lewis). Berkeley, University of California Press, 1918, 173

${ }^{83}$ Vgl. Zinov'ev.Folgebeziehung, 126-127.
} 
Arten (sinnvolle Sätze, Tautologien, Kontradiktionen) erzeugen, werden durch bejahende Operationen abgeschlossene Satzmengen erzeugt: Aus Tautologien gewinnt man Mengen von Tautologien, aus wahren sinnvollen Sätzen gewinnt man Mengen von wahren sinnvollen Sätzen ${ }^{84}$. Dieses Charakteristikum der bejahenden Operationen fällt mit dem Merkmal zusammen, das Tarski den Schlussregeln einer logischen Theorie zuspricht ${ }^{85}$. Die Regeln der Anwendung bejahender Operationen sind somit kein Bestandteil der Definition der allgemeinen Form des Satzes: Diese Definition ist aber die logische Voraussetzung der Definition der bejahenden Operationen. Das zeigt insbesondere die Rekonstruktion der Wittgensteinschen Definition der Wahrscheinlichkeit, die 1969 von von Wright vorgeschlagen wird $^{86}$. Diese Definition enthält nicht nur nach Meinung von Wrights problematischen Begriff der logischen Unabhängigkeit von Sätzen, sondern auch den Begriff der Wahrheitsfunktion, die gerade als Resultat einer Wahrheitsoperation definiert ist ${ }^{87}$. Folglich enthält die Definition einer Folgebeziehung zwischen Sätzen, die auf dem Begriff der Wahrscheinlichkeit gründet, und somit einer bejahenden Operation die Definition der Wahrheitsoperation und beruht auf dieser. Der Ausdruck der Beziehung zwischen Operationen könnte deshalb lauten: Ist auf ein syntaktisches Gebilde eine bejahende Operation nicht anwendbar, ist das fragliche Gebilde kein Resultat der Anwendung einer Wahrheitsoperation.

Trotz der angedeuteten Problematik teilen auch die bejahenden Operationen Wittgensteins die meisten Merkmale symbolischer Operationen der algebraisch-logischen Tradition. Sie sind auch Handlungen, die als ihre

\footnotetext{
${ }^{84}$ Die Möglichkeit, aus Kontradiktionen Sätze aller Arten als Schlüsse zu gewinnen sowie aus sinnvollen Sätzen Tautologien zu folgern, kann als ein definitorisches Merkmal von Tautologien und Kontradiktionen betrachtet werden, nicht als die Möglichkeit, die sich in einer dem Entwurf Wittgensteins entsprechenden Beschreibung der Welt realisiert.

${ }^{85}$ Tarski charakterisiert 1930 und 1935 Schlussregeln wie modus ponens als Operationen, welche abgeschlossene Systeme von Sätzen erzeugen. A. Tarski. „Fundamentale Begriffe der Methodologie der deduktiven Wissenschaften I“ (im weiteren:

Tarski.Begriffe). In: Monatshefte für Mathematik und Physik 37, 1930, 361-404, 363364, A. Tarski. „Grundzüge des Systemenkalküls“. In: Fundamenta Mathematicae XXV, 1935, 503-526, 507

${ }^{86}$ G.H. von Wright. "Wittgenstein on Probability". In: G.H. von Wright. Wittgenstein. Oxford, Basil Blackwell, 1982, 137-162, 141

${ }^{87}$ TLP, 5.234
} 
Basen und Resultate Sätze haben. Ihre Resultate können als ihre eigenen Basen auftreten, sofern ein bejahter Satz selbst einen anderen Satz bejahen kann. Als sinnvolle Sätze sind Resultate der bejahenden Operationen Wahrheitsfunktionen, die eine teilweise strukturelle Übereinstimmung mit ihren Basen aufweisen und deren Wahrheitsgründe die Wahrheitsgründe ihrer Basen enthalten. Der Zweck der bejahenden Operationen ist es, zu zeigen, welche Schlüsse in bezug auf Tatsachen aus einer bestimmten Tatsache folgen. Verneinende Operationen zeigen, welche Tatsachen durch eine beschriebene Tatsache ausgeschlossen sind. Das Problem dieses Zeigens besteht darin, dass durch die Anwendung einer bejahenden Operation das syntaktische Äquivalent des zu dieser Anwendung berechtigenden Zusammenhangs zwischen Tatsachen in dem Satzzeichen des Resultats der Operation aufgehoben werden kann. Die Anwendung des modus ponens besteht z.B. darin, dass man das Antezedens einer Implikation abtrennt, womit auch das Implikationszeichen eliminiert wird und als Folge der Anwendung der Regel nur das Konsequens der Implikation bleibt. Während ein Satzzeichen durch die Anwendung der Operation der Bejahung aus dem strukturellen Zusammenhang mit anderen Satzzeichen gelöst wird, wird der strukturelle Zusammenhang der Tatsachen, der in diesem Fall durch einen logischen Satz gezeigt wird, nicht zerstört: Er ist die Bedingung, ohne welche das logische Schließen nicht realisiert werden kann. Solche Zusammenhänge werden erst durch die logischen Sätze, d.h. Tautologien, gezeigt. Wenn also das Substrat der konstruktiven Operationen mit einer Weltbeschreibung identifiziert werden kann, ist das Substrat der bejahenden Operationen eine logische Theorie in Form einer Gesamtheit logischer Sätze. Die Gesetze, welche die Anwendung bejahender Operationen regeln, sind logische Schlussregeln, die nach Wittgenstein Symbole in dem Sinn sind ${ }^{88}$, dass sie in Gestalt von logischen Sätzen gegeben sind.

${ }^{88}$ Ebd., 5.514 
$\S 2$. Das logische Schließen und der Begriff des Sinnes

Betrachtet man die Bejahung als eine interne durch Symbole, nämlich Tautologien, gezeigte Beziehung zwischen Sätzen, kann man einen gegebenen Satz als Element des Feldes zweier Beziehungen betrachten: Eine Beziehung besteht zwischen dem gegebenen Satz und den Sätzen, die von ihm bejaht werden, eine andere Beziehung zwischen dem gegebenen Satz und den Sätzen, die ihn bejahen.

Die Beziehung des Satzes zu seiner Verneinung unterscheidet sich von seiner Beziehung zu jedem Satz, der ihn bejaht oder von ihm bejaht wird: Die Verneinung liegt ,ganz außerhalb“ des Satzes, während die anderen Sätze etwas mit $\mathrm{ihm}^{89}$ und miteinander gemein haben. Das Gemeinsame eines Satzes und der Sätze, die von ihm bejaht werden oder ihn bejahen, sind Wahrheitsgründe. Sie sind das, was der Satz und seine Verneinung miteinander nicht teilen: Der Satz und seine Verneinung können nicht beide zugleich wahr sein, sofern sie keine Wahrheitsmöglichkeiten aufweisen, welche die beiden Sätze zugleich bewahrheiten.

Die Folgebeziehung zwischen zwei Sätzen, die durch das Maß der Wahrscheinlichkeit, das man aus dem Verhältnis der Anzahlen der Wahrheitsgründe des als Prämisse des Schlusses auftretenden Satzes und seiner mit dem Schlusssatz gemeinsamen Wahrheitsgründe errechnet, charakterisiert wird, kennzeichnet auch die Beziehung zwischen dem Sinn eines der Sätze (oder dem, wie viel er sagt) und dem Sinn des anderen ${ }^{90}$. Sofern die Folgebeziehungen durch Berechnung bestimmt werden können, dienen sie selbst als Maß des Sinnes eines Satzes. Deswegen erfüllt Wittgensteins Auffassung des logischen Folgens auch die Funktion, sinnvolle Sätze als einzig mögliche Komponenten einer Weltbeschreibung von Tautologien und Kontradiktionen zu unterscheiden.

Elementarsätze zusammen mit allen aus ihnen konstruierbaren Sätzen bilden nach Wittgenstein einen logischen Raum. Diesen Begriff kann man nicht nur als eine Metapher betrachten ${ }^{91}$, sondern auch als einen Begriff, der den Begriff einer möglichen Weltbeschreibung erklärt. So bietet Stenius (1960), der den logischen Raum als einen unendlich-dimensionalen (der

\footnotetext{
${ }^{89}$ Ebd., 5.513

${ }^{90}$ Ebd., 5.122, 5.14

${ }^{91}$ S. z.B. Black.Companion, 10, 154.
} 
Anzahl der Elementarsätze entsprechend) zweiwertigen Ja-und-Nein-Raum beschreibt, sogar eine anschauliche Darstellung für diesen Raum ${ }^{92}$. Der Unterschied zwischen den drei Satzarten lässt sich am besten zeigen, wenn man den trivialen Fall eines durch einen einzigen Elementarsatz bestimmten eindimensionalen logischen Raums betrachtet. Ein solcher Raum lässt sich durch logische Beziehungen zwischen vier Satzzeichen darstellen (Schema 2). Dass ein Satz von einem anderen bejaht wird oder aus ihm logisch folgt, wird auf dem Schema durch einen Pfeil dargestellt, der vom bejahenden zum bejahten Satz (von der Prämisse zum Schluss) gerichtet ist. Aus einer Kontradiktion ,(FF)(p)“, die insbesondere die Gestalt ,p. $\sim p^{“}$ annehmen kann, folgt sowohl der Satz ,,(WF)(p)“ (der Satz ,p“) als auch der Satz „,(FW)(p)“ (der Satz , $\sim p^{“}$ ). Aus diesen beiden Sätzen folgt nur die Tautologie ,(WW)(p)“. Die Kontradiktion erfüllt den logischen Raum in dem Sinn, dass aus ihr ein jeder Satz, der in diesem Raum konstruierbar ist, logisch folgt. Die Tautologie ist die Grenze des Raums, sofern aus ihr kein sinnvoller Satz logisch folgen kann.

Ein sinnvoller Satz kann im Unterschied zu Kontradiktionen und Tautologien den logischen Raum in Spielräume teilen. Den Spielraum eines Satzes kann man als Bereich von Tatsachen verstehen, für welche die Wahrheitsmöglichkeiten des Satzes den Satz bewahrheiten ${ }^{93}$. Das logische Folgen, das eine Verengung des Sinnes ergibt, resultiert laut dieser Auffassung in einer Erweiterung des Spielraums, sofern die Wahrheitsgründe des Schlusssatzes die Wahrheitsgründe eines ihn bejahenden Satzes einschließen. Der Spielraum des Satzes, der aus einem anderen folgt, umfasst somit den Spielraum seiner Prämisse, und der Spielraum eines jeden Satzes liegt außerhalb des Spielraums seiner Verneinung. Eine Frage, die einer sich stellen kann, ist, ob und wann Spielräume zweier Sätze sich schneiden können ${ }^{94}$. Welche Bedingung zwei solche Sätze erfüllen müssen, zeigt das Schema 3. Die Konjunktion von $\mathrm{p}$ und $\mathrm{q}$ (der Satz ,(WFFF)(p,q)“) sowie

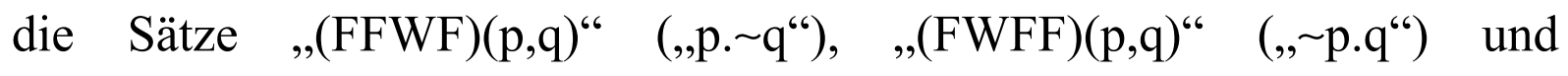

\footnotetext{
92 S. Stenius.Tractatus, 46, 52. Ich werde im weiteren diese Auffassung gebrauchen, obwohl ich die Darstellungsweise Stenius' bei dem Versuch, die Folgebeziehungen zu veranschaulichen, nicht übernehmen werde.

${ }^{93}$ F. Waismann. „Logische Analyse des Wahrscheinlichkeitsbegriffs“. In: Erkenntnis 1, 1930/1931, 228-248, 235-236.

${ }^{94}$ Ebd., 236
} 
,(FFFW)(p,q)“ (,, p p $\left.\sim q^{c *}\right)^{95}$ sind alternative Weltbeschreibungen, die in einem zweidimensionalen Ja-und-Nein-Raum möglich sind ${ }^{96}$. Jedem der Satzzeichen Wittgensteins entsprechen unterschiedliche mit den Mitteln der traditionellen aussagenlogischen Notation konstruierbare Wahrheitsfunktionen von den Argumenten-Paaren $\mathrm{p}$ und $\mathrm{q}, \sim \mathrm{p}$ und $\mathrm{q}, \mathrm{p}$ und $\sim \mathrm{q}, \sim \mathrm{p}$ und $\sim$ q. So entsprechen dem Satz ,(WFFF)(p,q)“ die Sätze ,p.q.", ,p $\not \sim q “$, ,$\sim p \not \subset q^{\prime \prime}$ und , $\sim \mathrm{p} \mid \sim \mathrm{q}^{\prime \prime}$. Dieses Entsprechen bedeutet, dass es für ein Paar von Elementarsätzen und eine gewisse Gruppe von Wahrheitsbedingungen in der traditionellen Notation ${ }^{97}$ vier verschiedene Satzzeichen konstruierbar sind. Ableitungen in den alternativen Weltbeschreibungen, d.h. Beziehungen zwischen den Weltbeschreibungen und Wahrheitsfunktionen, die aus jeder der Weltbeschreibungen logisch folgen, sind auf dem Schema durch unterschiedlich gestrichene Linien dargestellt. Dem Schema kann man entnehmen, dass sich z.B. die Spielräume der Sätze „,(WFFW)(p,q)“

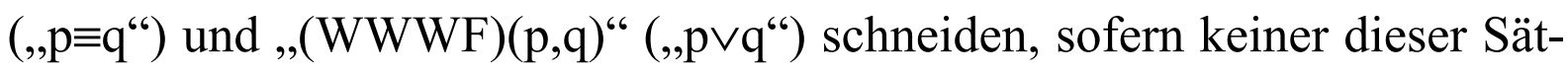
ze aus dem anderen folgt, die beiden jedoch aus der Konjunktion von $\mathrm{p}$ und q logisch folgen und deswegen beide den Spielraum dieses Satzes umfassen (Schema 4).

Des weiteren zeigt das Schema 3, dass der Satz ,(FWWF)(p,q)“ (,p年q“) einerseits den Satz ,(FWWW)(p,q)“, d.h. die Verneinung der Konjunktion von $\mathrm{p}$ und $\mathrm{q}$, und andererseits die Disjunktion von $\mathrm{p}$ und $\mathrm{q}$ (den Satz „(WWWF)(p,q)“), die aus der Konjunktion dieser Sätze logisch folgt, bejaht. Das bedeutet, dass sich die Spielräume der Verneinung der Konjunktion und einer ihrer Folgen schneiden und deswegen eine logische Folge eines Satzes etwas Gemeinsames mit seiner Verneinung haben kann. Das Vorkommen solcher Schnittpunkte erklärt, warum der Spielraum eines Satzes durch die ihn bejahenden Sätze und nicht durch die Sätze, die von

\footnotetext{
${ }^{95}$ Bei der Verteilung der Abzeichen „W“ und „F“ folge ich der Verteilung Wittgensteins. Vgl. TLP, 5.101.

${ }^{96}$ Dieselben Weltbeschreibungen werden von Stenius analysiert. Stenius. Tractatus, 54-55

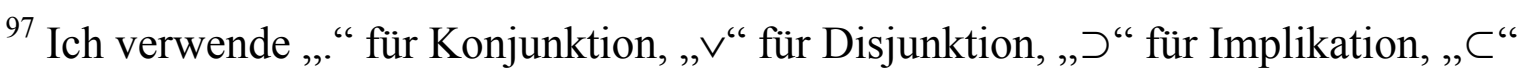
für konverse Implikation, „, “ für Äquivalenz, „, “ für Antikonjunktion, ,,|“ der Notation Wittgensteins entsprechend für Antidisjunktion, , $\not \supset$ “ für Antiimplikation, „ $\not \subset$ “ für konverse Antiimplikation und „,\#“ für Antiäquivalenz.
} 
ihm bejaht werden, definiert wird: Der Spielraum eines Satzes liegt deswegen außerhalb des Spielraums seiner Verneinung, weil der Satz aus keiner Prämisse ${ }^{98}$ seiner Verneinung logisch folgen kann.

Das Schema 3 verdeutlicht, warum Wittgenstein von Sheffers Notation als von der Erfindung Sheffers spricht. Den Sinn dieser Erfindung sieht Wittgenstein darin, dass die Notation Sheffers ,die nötige Multiplizität hat ${ }^{\text {“99 }}$. Diese Behauptung lässt sich dadurch erklären, dass einer jeden Menge aus vier dieselben Elementarsätze enthaltenden und die gleichen Wahrheitsbedingungen aufweisenden Satzzeichen in der üblichen Notation nur ein Satzzeichen in der Notation Sheffers entspricht. Auf dem Schema ist ein solches Zeichen direkt unter dem Satzzeichen in der Notation Wittgensteins angegeben.

Der Spielraum des Satzes ist nach Stenius zugleich der logische Ort des Satzes, der nicht ein Punkt des logischen Raums ist, sondern durch eine Menge von Punkten oder Sätzen gegeben ist. Diese Menge ist laut Stenius die Menge der alternativen Weltbeschreibungen, die mit dem fraglichen Satz in dem Sinn verträglich sind, dass der Satz in diesen Weltbeschreibungen wahr ist ${ }^{100}$. Wenn man nun die Folgebeziehungen zwischen Sätzen in Betracht zieht, kann man, von der These Wittgensteins ausgehend, dass der verneinende und der verneinte Satz verschiedene logische Orte haben ${ }^{101}$, den logischen Ort des Satzes als Menge der Sätze betrachten, die den Satz bejahen. Die Sätze dieser Menge sind mit dem Satz insofern verträglich, als die Verneinung des Satzes die Verneinung eines jeden ihn bejahenden Satzes zur Folge hat. Deswegen kann man den logischen Ort des Satzes mit dem Spielraum identifizieren, der vom Satz den Tatsachen gelassen wird. Diese Übereinstimmung resultiert daraus, dass der logische Ort eines gegebenen Satzes dann bestimmt ist, wenn Elementarsätze, aus welchen der Satz konstruiert ist, und somit die Wahrheitsbedingungen des

\footnotetext{
${ }^{98}$ Unter Prämisse eines Schlusses verstehe ich dabei, Wittgenstein folgend, nur einen sinnvollen Satz, keine Kontradiktion.

${ }^{99}$ L. Wittgenstein. „IV. Philosophische Bemerkungen“ (im weiteren:

IV.Bemerkungen). In: L. Wittgenstein. Wiener Ausgabe 2. Wien, New York, Springer 1994, 205-333, 229

100 Stenius. Tractatus, 55-57

101 TLP, 4.0641
} 
Satzes, die zum Satzzeichen gehören und die man auch als Bestandteile der logischen Koordinaten des Satzzeichens auffassen kann, gegeben sind ${ }^{102}$. Die Analyse der Beziehungen des Bejahens zeigt Folgendes. Die Auffassung der logischen Konstanten als Zeichen, die nicht vertreten, sowie der Satzzeichen als Symbole, die zusammen mit Zeichen von Elementarsätzen auch Abzeichen für Wahrheitsbedingungen mitführen, garantiert, dass Sätze, welche aus denselben Elementarsätzen bestehen und dieselben Wahrheitsbedingungen aufweisen, denselben Spielraum bestimmen und deswegen denselben Sinn haben. Folglich ergibt das deduktive logische Schließen einen neuen, und zwar verengten, Sinn in den Fällen, in welchen der Schluss durch eine nicht umkehrbare logische Implikation ermöglicht wird.

\section{$\S 3$. Der logische Symbolismus und die logische Theorie}

Logische Operationen sind für Wittgenstein Instrumente der Konstruktion des Symbolismus und seiner Anwendung. Sofern im Tractatus in erster Linie die Prinzipien der Konstruktion des Symbolismus formuliert und begründet werden, stellt sich die Frage, wie sich die Konstruktion dieser Art zur Konstruktion einer logischen Theorie verhält.

Wittgenstein setzt die Angabe einer genügenden Notation mit der Angabe der logischen Wahrheiten (logischen Sätze) gleich und erklärt die Methode der logischen Theorien, welche die Formulierung der logischen Sätze mit den Beweisen dieser Sätze verknüpfen, für unwesentlich für die Logik ${ }^{103}$. Das von solchen Theorien angestrebte Beweisen der logischen Sätze, das in der Anwendung logischer Operationen auf wenige ausgezeichnete Symbole und in ihrer Transformation besteht, wobei die Rolle solcher transformierbarer Symbole Axiome oder logische Grundgesetze erfüllen, ist aus dem Grund unwesentlich, dass die Logik ihre Grundgesetze ohne Beweis anerkennt. Werden die Sätze durch Abzeichen für Wahrheitswerte ergänzt, wird das Beweisen unnötig und die Bestimmung der logischen Grundgesetze entbehrlich ${ }^{104}$, sofern die logischen Sätze von anderen Sätzen durch ihren tautologischen Charakter unterschieden werden. Dass Wittgenstein die Beziehungen zwischen logischen Operationen und Beweisen in der

\footnotetext{
${ }^{102}$ Ebd., 4.431, 3.4-3.41

${ }^{103}$ Ebd., 6.1223, 6.126

${ }^{104}$ Vgl. ebd., 6.127-6.1271.
} 
Logik als die durch „bloße Zeichenregeln“ gegebenen Beziehungen deutet, zeigt, dass er logische deduktive Systeme als Zeichensysteme behandelt: Die Elemente eines Zeichensystems sowie ihre Beziehungen werden durch bestimmte syntaktische Konventionen geregelt, wobei die Anwendung solcher Konventionen sich letztendlich an die Bestimmung und Veränderung der Gestalt der Elemente des Zeichensystems richtet.

Im Tractatus wird eine Alternative zu einer axiomatischen logischen Theorie entworfen. Diese Alternative ist durch drei Charakteristika bestimmt. Erstens werden logische Operationen als syntaktische Operationen charakterisiert. Dieses Charakteristikum teilt eine dem Entwurf Wittgensteins entsprechende Konstruktion mit einer dem Ideal der formalen Reinheit entsprechend axiomatisch aufgebauten logischen Theorie. Eine solche Theorie bezeichnet man als einen Kalkül, den man als ein formales oder syntaktisches System auffasst und als System von Regeln angibt. Zweitens werden durch die Einführung der Wahrheitswerte-Abzeichen einige die Anwendbarkeit der logischen Theorie bestimmende Regeln der semantischen Interpretation von Symbolen zum Bestandteil der Symbole (der Satzzeichen) selbst $^{105}$. Dadurch wird ein Teil der Gesamtheit semantischer Regeln, die üblicherweise zusammen mit dem syntaktisch aufgebauten formalen vom logischen Schließen handelnden System eine interpretierbare logische Theorie ergeben, zum Bestandteil der Syntax, d.h. des formalen Systems selbst. Drittens werden logische Sätze als eine aus Tautologien bestehende Teilmenge der Menge der im Symbolismus konstruierbaren Sätze definiert. Obwohl diese Definition mit dem Verzicht auf die Konstruktion der Beweise der logischen Sätze einhergeht, widerspricht die Auffassung Wittgensteins nicht den Prinzipien der Beweiskonstruktion in der Logik. Wenn

${ }^{105}$ Dieses Charakteristikum der Auffassung Wittgensteins wird besonders deutlich von Th. Hailperin unterstrichen. S. Th. Hailperin. „Foundations of Probability in Mathematical Logic". In: Philosophy of Science 4, 1937, 125-150, 127-128. Dieses Charakteristikum wird oft außer acht gelassen, was manche Autoren zu Behauptungen veranlasst, dass für das auf den Beziehungen des logischen Folgens beruhende Schließen allein die Struktur der Prämissen und des Schlusssatzes verantwortlich ist und dass die Berechnung des Wahrscheinlichkeitsmaßes und das Heranziehen der Wahrheitsgründe der Sätze als anschauliche Hilfestellung für diejenigen gedacht ist, die sich die Notation noch nicht angeeignet haben. S. z.B. I. Proops. Logic and Language in Wittgenstein's Tractatus. New York, London, Garland Publishing, Inc., 2000, 89-91. Die Aneignung der Notation wird somit als Aneignung einer bestimmten Technik (wie in den Philosophischen Untersuchungen) behandelt. 
Beweisoperationen durch logische Gesetze in Form von Regeln gegeben sind, kann man Regeln als Ersatz für Axiome eines formalen Systems behandeln, sofern Regeln eine Menge von logischen Sätzen auf einem zur Angabe eines axiomatischen Systems alternativen Weg bestimmen. Die Idee einer solchen Auffassung von Regeln äußert 1929 Hertz, der behauptet, dass als Axiome, die ein abgeschlossenes System von Sätzen definieren, Verknüpfungsregeln fungieren können, wobei ein System von Sätzen dann abgeschlossen ist, wenn die Konklusion eines jeden Schlusses, dessen Prämissen zum System gehören, ebenfalls zum System gehört. Regeln geben an, wie man aus Prämissen eine Konklusion findet, die mit Prämissen zusammen einen Schluss bildet ${ }^{106}$. Die Möglichkeit dieser Alternative erklärt auch die These Wittgensteins, dass jeder logische Satz sein eigener Beweis ist ${ }^{107}$, sofern sich für diese These eine entsprechende Parallele in den Systemen des natürlichen Schließens zeigt. Die Besonderheit der Methode solcher Systeme besteht in der Herleitung von Sätzen aus Annahmen $^{108}$ : Die Gültigkeit einer Implikation wird gezeigt, indem gezeigt wird, dass das Konsequens der Implikation aus ihrem Antezedens herleitbar ist $^{109}$. Die These Wittgensteins kann als Definition einer semantischen Variante dieses Verfahrens betrachtet werden, sofern er die Herleitung durch die Berechnung der Wahrheitsbedingungen der Prämisse und des Schlusssatzes des Schlusses ersetzt.

Während Wittgenstein der Konstruktion der Logik als einer Beweistheorie skeptisch gegenübersteht, ist für ihn das logische Beweisen sinnvoller Sätze einer der Zwecke, welchen der Aufbau des logischen Symbolismus verfolgt. Das Beweisen sinnvoller Sätze mittels anderer sinnvoller Sätze fasst er als logisches Folgern auf, das durch logische Sätze geregelt ist. Von seinen Thesen ausgehend, kann man einen Satz als bewiesen betrachten, wenn seine Wahrheit aus der Wahrheit eines anderen Satzes nach einem logischen Satz folgt ${ }^{110}$. Zum Zweck des Symbolismus gehört somit nach

\footnotetext{
${ }^{106}$ P. Hertz. „Über Axiomensysteme für beliebige Satzsysteme“. In: Mathematische Annalen 101, 1929, 457-514, 461, 465

107 TLP, 6.1265

${ }^{108}$ Gentzen.Untersuchungen, 184

109 S. z.B. W.V. Quine. "On Natural Deduction”. In: The Journal of Symbolic Logic $15,1950,93-102,93-94$.

${ }^{110}$ Vgl. TLP, 5.12, 6.1263-6.1264.
} 
Wittgensteins Ansicht, die logischen Folgebeziehungen zwischen sinnvollen Sätzen zu begründen.

$\S 4$. Logische Theorie Wittgensteins und die Entwicklung der Logik im 20ten Jahrhundert

Sofern Wittgenstein den logischen Symbolismus von einer in der Form eines symbolischen Systems aufgebauten logischen Theorie unterscheidet, stellt sich die Frage, wie sich seine Theorie zu der Entwicklung der Logik im 20ten Jahrhundert verhält. Diese Frage ist um so dringlicher, als diese Entwicklung durch die Darstellung logischer Theorien als symbolischer Systeme gekennzeichnet ist. Die Analyse des Operationsbegriffs Wittgensteins zeigt, dass seiner Theorie die Rolle einer derjenigen logischen Theorien gebührt, welche die Vereinigung der algebraisch-logischen Methoden einerseits und der auf das Fregesche Ideal der Begriffsschrift zurückgehenden Prinzipien der mathematischen Logik andererseits und somit die Entwicklung der Metalogik einleiten.

\section{$\S 4 a$. Logik als ein Kalkül}

Wenn man die Logik als einen Kalkül charakterisiert, kann diese Charakterisierung unterschiedlich verstanden werden.

Einerseits versteht man unter einem Kalkül eine logische Theorie, die als ein formales oder syntaktisches System aufgebaut wird. Bei einem solchen Aufbau wird die Logik durch die Definition einer besonderen formalen Sprache als ein System von Regeln eingeführt.

Andererseits versteht man unter einem Kalkül ein nicht rein formal aufgebautes System von Prinzipien, die eine Ableitungstechnik als eine Gesamtheit algebraisch-logischer Beziehungen definieren.

Die Unterscheidung zwischen diesen Auffassungen benutzt 1967 van Heijenoort, um zwei verschiedene sich in den 20er Jahren des 20ten Jahrhunderts vereinigende Strömungen in der Entwicklung der Logik seit Peirce, Schröder und Frege zu charakterisieren. Die Logik, die als ein formales 
System konstruiert wird, bezeichnet van Heijenoort als eine Sprache. Eine algebraisch-logische Theorie bezeichnet er als einen Kalkül ${ }^{111}$.

Die Bezeichnungsweise van Heijenoorts lässt sich auf die Unterscheidung zurückführen, welche Frege macht, wenn er seine Begriffsschrift als lingua characteristica im Unterschied zum Booleschen calculus ratiocinator definiert. Dass Frege seine Logik der Booleschen auf diese Weise entgegensetzt, erklärt sich dadurch, dass Frege seine Aufgabe in der Formulierung der Sprache sieht, die für die wissenschaftlichen Zwecke und insbesondere für die Begründung der Mathematik besser geeignet ist als die Alltagssprache. Deswegen ist das, was er entwickelt, eine Sprache, die nach streng formulierten und ohne Ausnahmen geltenden Regeln wie ein Werkzeug oder eine künstliche Hand arbeitet ${ }^{112}$, mit anderen Worten ein System von Regeln, das auch als eine formale Sprache oder ein Kalkül bezeichnet wird.

Das Wesentliche der Unterscheidung zwischen Logik als einer Sprache und Logik als einem Kalkül beschreibt van Heijenoort auch als Gegensatz zwischen der von Frege sowie Russell und Whitehead entwickelten universalen Logik und der von Peirce, Schröder und später von Löwenheim vertretenen metasystematischen Herangehensweise an die Formulierung der logischen Theorie. Universale Logik lässt keinen Wechsel der Diskussionsbereiche zu und verzeichnet als ihren einzigen Diskussionsbereich das Universum, zu welchem auch abstrakte Gegenstände gehören, und welches nach Russell und Whitehead in Typen eingeteilt ist ${ }^{113}$. Das Universum, das von van Heijenoort dieser Logik unterstellt wird, ist somit das Universum aller möglichen Gegenstände des Denkens, welchen man etwas prädizieren und die man in Beziehungen zu einander setzen kann. In den Arbeiten der Vertreter der metasystematischen Strömung werden Ergebnisse laut van Heijenoort meistens durch semantische Betrachtungen und nicht durch formale syntaktische Konstruktionen erreicht. Deswegen bezeichnet van Heijenoort die universale Logik auch als syntaktische Strömung und die

\footnotetext{
111 J. van Heijenoort. "Logic as Calculus and Logic as Language" (im weiteren: van Heijenoort.Logic). In: J. van Heijenoort. Selected Essays. Napoli, Bibliopolis, 1985, $11-16,12$

${ }^{112}$ G. Frege. "Ueber die wissenschaftliche Berechtigung einer Begriffschrift". In: I. Angelelli (Hrsg.). G. Frege. Begriffschrift und andere Aufsätze. Darmstadt, Wissenschaftliche Buchgesellschaft, 1974, 3. Aufl., 106-114, 110

113 van Heijenoort.Logic, 12
} 
metasystematische Strömung als semantische Auffassung der Logik. Diese letzte Bezeichnungsweise entspricht der von Hermes und Scholz 1952 gegebenen Charakteristik der Syntax und Semantik durch die ihre Fragestellungen bestimmenden Begriffe ${ }^{114}$.

Einer der Zwecke der Unterscheidung zwischen den beiden Strömungen ist die Begründung ihrer Tendenz zur Vereinigung ${ }^{115}$. Nur diese Vereinigung kann nach Meinung Drebens und van Heijenoorts (1986) zugleich den Aufbau eines formalen logischen Systems und die Formulierung der metasystematischen Fragen nach den Eigenschaften eines solchen Systems, wie der Frage nach seiner Vollständigkeit, ermöglichen. Die Frege-Russellsche Strömung ist unfähig, solche Fragen wegen der Universalität ihrer logischen Systeme als Probleme zu erkennen und folglich sie zu formulieren. Vertreter der Gegenströmung wie Löwenheim können solche Fragen nicht stellen, weil bei ihnen ein formales System mit solchen Komponenten wie Axiome und Regeln nicht aufgebaut wird ${ }^{116}$.

Der Unterschied zwischen den beiden Strömungen oder Herangehensweisen an die Logik - der Algebra der Logik einerseits und der von Frege eingeleiteten Tradition andererseits - ist in der Geschichtsschreibung der Logik anerkannt. Bocheński (1956) charakterisiert diesen als Unterschied von Perioden in der Entwicklung der mathematischen oder symbolischen Logik, dem zugrunde folgender inhaltlicher Unterschied liegt. Während die Boolesche Periode (1847-1895) dadurch gekennzeichnet ist, dass die mathematischen Methoden in den Werken ihrer Vertreter nicht als Gegenstand der Untersuchung auftreten, sondern auf die Logik als Mittel der logischen Untersuchung angewandt werden, ist das Hauptmerkmal der Fregeschen Periode (1879-1910/1913) die Beschäftigung mit der Begründung der Mathematik und die Entwicklung eines logischen objektsprachlichen Systems, in welchem eine Reihe von mathematisch-logischen Sätzen aus

${ }^{114}$ H. Hermes, H. Scholz. Mathematische Logik. Enzyklopädie der mathematischen Wissenschaften mit Einschluss ihrer Anwendungen 1-1-1. Leipzig, B.G. Teubner Verlagsgesellschaft, 1952, 3

115 J. van Heijenoort. „Set-Theoretic Semantics”. In: J. van Heijenoort. Selected Essays. Napoli, Bibliopolis, 1985, 43-53, 45-46

${ }^{116}$ B. Dreben, J. van Heijenoort. „Introductory Note to 1929, 1930 and 1930a”. In: S. Feferman, J.W. Dawson, Jr., S.C. Kleene, G.H. Moore, R.M. Solovay, J. van Heijenoort (Hrsg.). K. Gödel. Collected Works I. New York, Oxford, Oxford University Press, 1986, 44-59, 44-46 
wenigen Axiomen lückenlos abgeleitet ist ${ }^{117}$. In Hinblick auf diese Unterscheidung wäre es vielleicht sinnvoll, die universale Logik als ,intrasystematische" oder "begriffsschriftliche“ Strömung und die metasystematische Behandlung der Logik als „metasystematische“ oder ,algebraischlogische" Strömung zu charakterisieren.

Bocheński bezeichnet die von Dreben und van Heijenoort als metasystematische gekennzeichneten Fragen als metalogische. $\mathrm{Zu}$ Bereichen der Metalogik zählt er vor allem die von Tarski entwickelte Methodologie der deduktiven Systeme und die von Carnap entwickelte Syntax. Das Vorrükken der Metalogik, das Bocheński mit der Zeit nach 1910 datiert, fällt zeitlich mit der Entwicklung verschiedenster objektsprachlicher logischer Systeme zusammen, was erlaubt, die Metalogik als eine Art des theoretischen Begreifens dieser Entwicklung anzusehen. Dieses Vorrücken führt nach Meinung Bochénskis schließlich zur formalistischen Systematisierung der Metalogik selbst (die ungefähr 1930 anfängt) und zum Entstehen der Systeme, insbesondere der Systeme Gentzens und Jaśkowskis, in welchen Logik und Metalogik zusammengefasst werden ${ }^{118}$.

Die Tendenz zur Vereinigung der beiden Strömungen zeichnet sich besonders deutlich in einigen Arbeiten ab, z.B. 1918 in der Arbeit Lewis' und 1921 in der Arbeit Posts ${ }^{119}$. Bei der Untersuchung der Beziehungen zwischen der symbolischen Logik und Logistik bemerkt Lewis, dass eines der Charakteristika der eine besondere logistische Methode repräsentierenden Principia Mathematica darin besteht, dass die Darlegung des formalen Systems explizit den Bezug zum begrifflichen Inhalt des Systems herstellt und dass die Regeln des Operierens mit Symbolen zum Teil in den Erklärungen der Bedeutung der Notation enthalten sind. Lewis äußert den Gedanken, dass die Fragen des Inhalts nicht eine interne Angelegenheit des Systems sein müssen, sondern im Zusammenhang mit der Anwendung des Systems betrachtet werden können, so dass als Ideal der Darlegung der symbolischen Logik die Darlegung betrachtet wird, die klar zwischen dem Inhalt und der Form des Systems unterscheidet. Die Methode des Errei-

\footnotetext{
117 J.M. Bocheński. Formale Logik. Freiburg, München, Verlag Karl Alber, 2002, 313315

118 Ebd., 315

${ }^{119}$ E.L. Post. "Introduction to a General Theory of Elementary Propositions" (im weiteren: Post). In: American Journal of Mathematics 43, 1921, 163-185
} 
chens dieses Ideals sieht Lewis darin, dass die Operationsregeln des Systems als Regeln der Manipulation mit den ohne Bezug auf ihre mögliche Bedeutung definierten Zeichen oder Zeichenketten eingeführt werden ${ }^{120}$. Post unterscheidet ausdrücklich zwischen den Sätzen der logischen Theorie der Principia und den Sätzen über diese Theorie (den Sätzen, welche Aussagen über alle Sätze dieser Theorie beinhalten) ${ }^{121}$. Post bezieht sich wie Wittgenstein auf die Arbeit Sheffers, der 1913 bei der Untersuchung der Systeme von Postulaten für Boolesche Algebren die Anzahl der primitiven logischen Konstanten reduziert, indem er eine neue Konstante einführt, die als Zeichen einer binären Kombinationsregel oder Funktion von zwei logischen Argumenten fungiert ${ }^{122}$. Der Bezug auf Sheffer dient Post zur Entwicklung der Methode einer metasystematischen Betrachtung, sofern dieser Bezug den Vergleich von verschiedenen „Wahrheitssystemen“ (Systemen der Sätze der Aussagenlogik, die aufgrund von bestimmten Axiomen und Operationen behauptet werden können und von Post als ,positive Funktionen“" bezeichnet werden) ermöglicht. Post zeigt, dass das mit dem Sheffer-Strich als einem primitiven Operator konstruierbare Wahrheitssystem ein vollständiges, dem Wahrheitssystem der Principia äquivalentes System ist ${ }^{123}$. Post bezeichnet die in der Aussagenlogik konstruierbaren syntaktischen Ausdrücke oder Formeln als Funktionen, die Ableitungsregeln als Operationen ${ }^{124}$, und benutzt die Behauptungen über Wahrheitswertetabellen, mit welchen man die Wahrheitswerte einer Funktion berechnet, als Mittel der Lösung des Problems der Beweisbarkeit für die Aussagenlogik der Principia.

Auch der Tractatus stellt ein besonderes Beispiel derselben Vereinigungstendenz dar. Wittgenstein formuliert einerseits die Forderung der Reinheit des logischen Symbolismus. Dass bei der Formulierung von Regeln und Einführung von Zeichen in dem Symbolismus der Principia Russell und Whitehead von Bedeutungen der Zeichen reden müssen und Definitionen und Grundgesetze in Worten (nicht in logischen Symbolen) formulieren,

${ }^{120}$ Lewis, 356-360
${ }^{121}$ Post, 164

${ }^{122}$ H.M. Sheffer. "A Set of Five Independent Postulates for Boolean Algebras, with Application to Logical Constants". In: Transactions of the American Mathematical Society 14, 1913, 481-488, 481-482

${ }^{123}$ Post, 173-174

${ }^{124}$ Ebd., 165-166 
betrachtet Wittgenstein als Fehler in der Formulierung einer logischen Notation $^{125}$. Obwohl man in einigen Besonderheiten der Wittgensteinschen Auffassung des Symbolismus sogar die Wurzeln von van Heijenoorts Terminus „universale Logik“ sehen kann $^{126}$ und Wittgenstein die metasystematischen Fragen nicht ausdrücklich formuliert, kann man seine Unterscheidung zwischen Tautologien, Kontradiktionen und sinnvollen Sätzen zusammen mit der Auffassung der Abzeichen für die Wahrheitswerte als Bestandteile der Satzzeichen als eine Methode der Lösung des metasystematischen Entscheidungsproblems für die Aussagenlogik auffassen. Die im Tractatus formulierten Prinzipien der Konstruktion und Anwendung eines logischen Symbolismus kann man mit einigen Prinzipien der Entwicklung der natürlichen Kalküle vergleichen, und schließlich den dem Entwurf Wittgensteins entsprechenden logischen Symbolismus in Hinblick auf seinen Zweck, die Schlussregeln für sinnvolle Sätze zu formulieren, auch als einen Kalkül im Sinne eines algebraisch-logischen Systems von Prinzipien, die eine Ableitungstechnik als eine Gesamtheit logischer Beziehungen definieren, charakterisieren.

Ermöglicht wird diese Auffassung der Logik erstens dadurch, dass Wittgenstein die Prinzipien der Konstruktion des Symbolismus als Regeln des Aufbaus einer besonderen Sprache behandelt, und zweitens durch die Ähnlichkeit seiner Auffassung der Operationen mit der Auffassung, welche die algebraisch-logische Strömung charakterisiert.

\footnotetext{
${ }^{125}$ TLP, 3.33-3.331, 5.452

${ }^{126}$ Die Forderung der Universalität der Logik, die universal als Mittel des Sprechens über die Welt ist, äußert sich in der Bildtheorie des Tractatus, die von der Gegebenheit der Elementarsätze und dem irreduziblen Charakter von Namen ausgeht. S. TLP, 4.51, 5.524, 5.557. Die Möglichkeit, die Elementarsätze und somit die Gegenstände der Welt in ihrer Gesamtheit zu erfassen (ebd., 5.524, 4.26-4.27), bedeutet, dass man in der Logik mit dem ganzen Universum der Gegenstände als mit der Voraussetzung der Logik zu tun hat (ebd., 5.552), zugleich aber aus diesem Universum nicht hinaus kann (ebd., 5.61). Der Symbolismus wird als ein System von Regeln konstruiert, der so zu sagen von außen, d.h. durch seine Beschreibung, nicht analysiert werden kann. Sieht man von der Natur der Gegenstände der Welt ab, oder erkennt man als diese auch abstrakte Gegenstände wie Klassen und Wahrheitswerte an, lässt sich die These über die Universalität der Logik auf die die Problematik des Tractatus verursachenden logischen Theorien übertragen, wobei durch diese Übertragung die Systeme Freges einerseits und Russells und Whiteheads andererseits als Quellen des Tractatus anerkannt werden.
} 


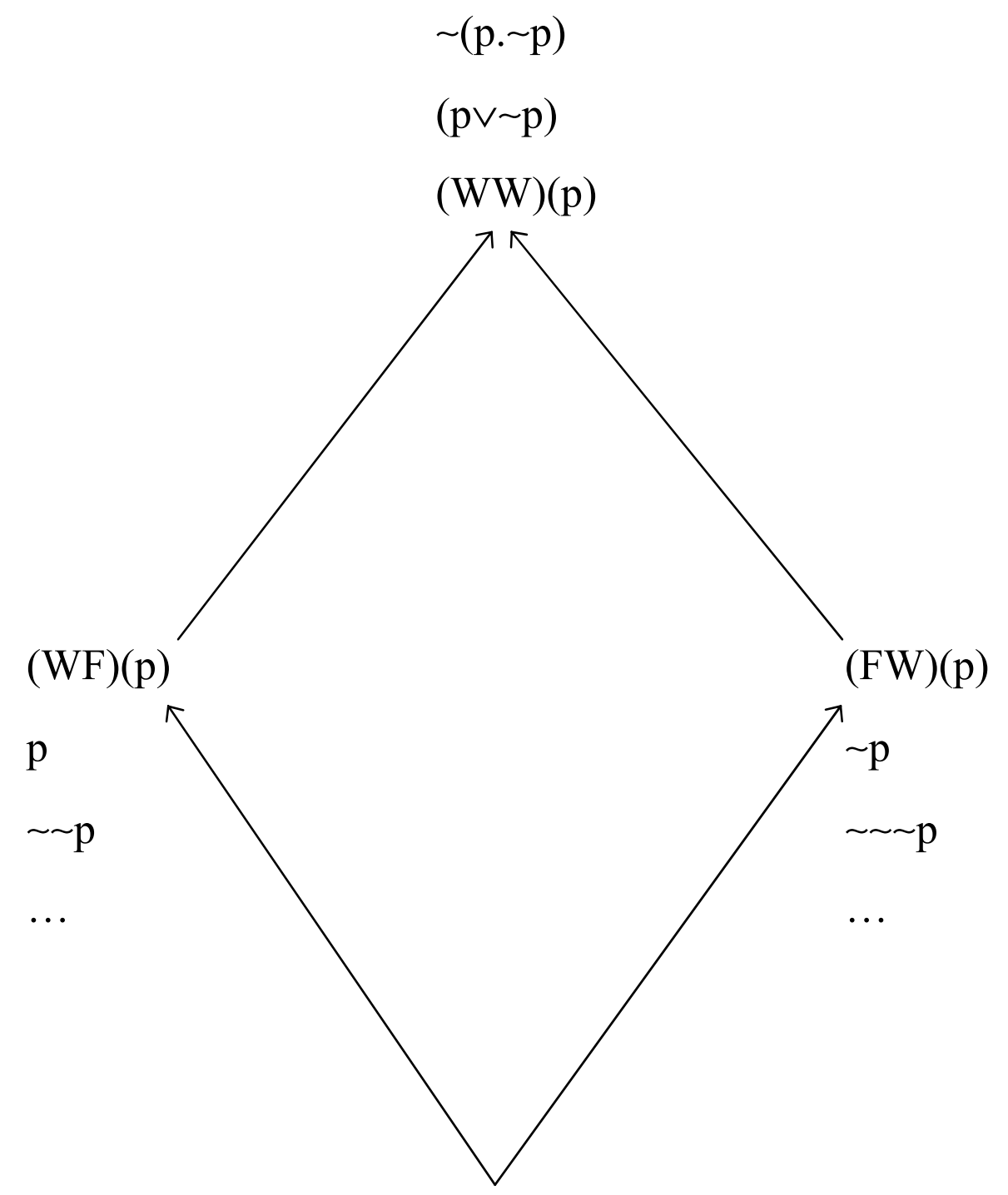

(FF)(p)

(p. $\sim p)$

Schema 2 


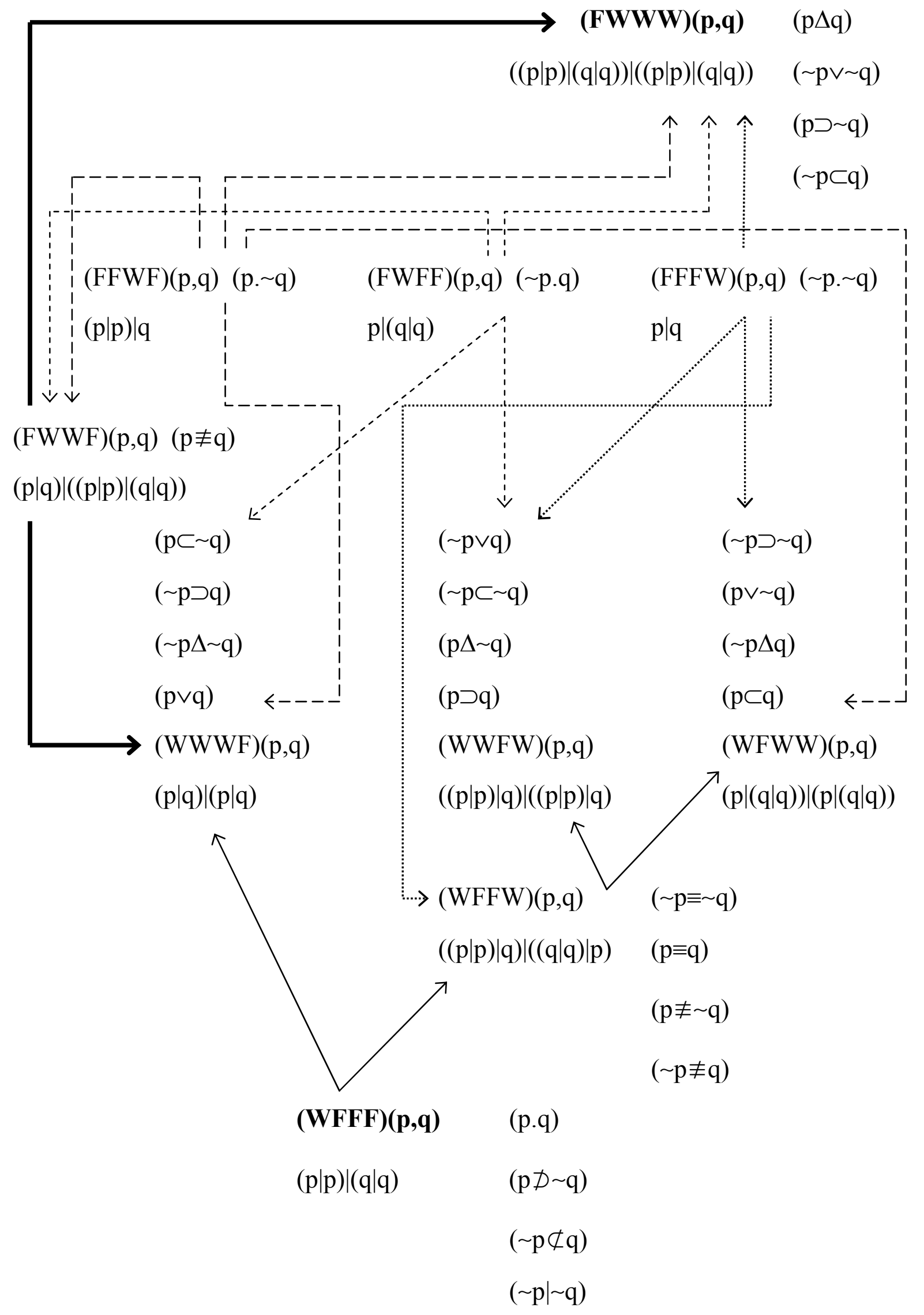

Schema 3 


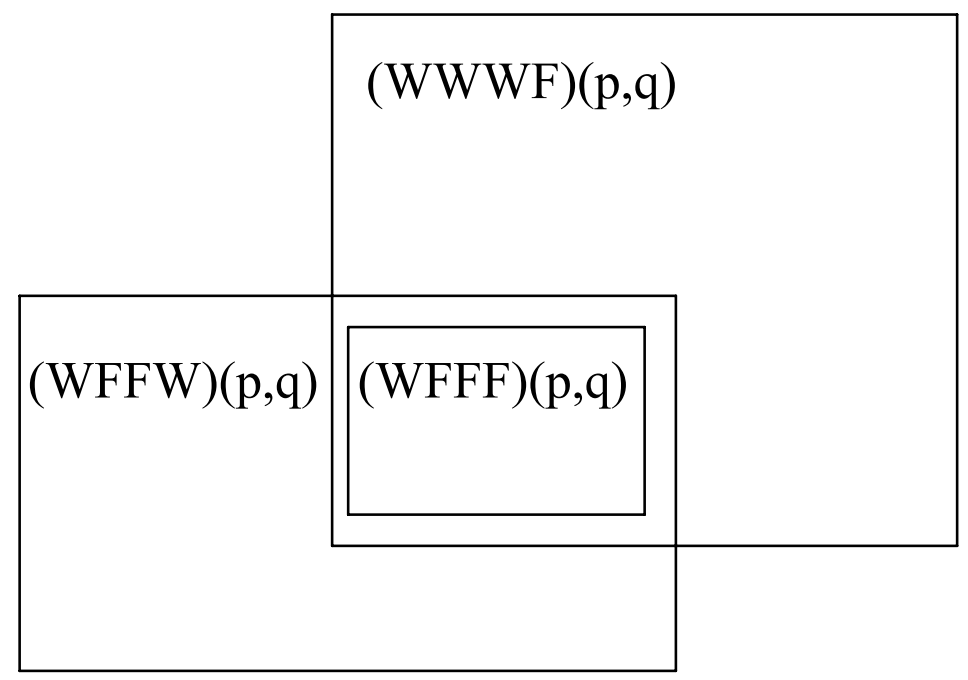


